Article

\title{
Composite and Background Fields in Non-Abelian Gauge Models
}

\author{
Pavel Yu. Moshin ${ }^{1, *}$ and Alexander A. Reshetnyak 1,2,3 $\mathbb{D}$ \\ 1 Department of Physics, National Research Tomsk State University, 634050 Tomsk, Russia; \\ reshet@tspu.edu.ru \\ 2 Center for Theoretical Physics, Tomsk State Pedagogical University, 634061 Tomsk, Russia \\ 3 Institute of Strength Physics and Materials Science Siberian Branch of Russian Academy of Sciences, \\ 634021 Tomsk, Russia \\ * Correspondence: moshin@phys.tsu.ru
}

Received: 19 October 2020; Accepted: 25 November 2020; Published: 30 November 2020

check for updates

\begin{abstract}
A joint introduction of composite and background fields into non-Abelian quantum gauge theories is suggested based on the symmetries of the generating functional of Green's functions, with the systematic analysis focused on quantum Yang-Mills theories, including the properties of the generating functional of vertex Green's functions (effective action). For the effective action in such theories, gauge dependence is found in terms of a nilpotent operator with composite and background fields, and on-shell independence from gauge fixing is established. The basic concept of a joint introduction of composite and background fields into non-Abelian gauge theories is extended to the Volovich-Katanaev model of two-dimensional gravity with dynamical torsion, as well as to the Gribov-Zwanziger theory.
\end{abstract}

Keywords: composite fields; background fields; Yang-Mills theory; Volovich-Katanaev model; Gribov-Zwanziger model; background effective action; gauge-dependence

\section{Introduction}

Composite [1,2] and background [3-5] fields are widely used in quantum gauge theories. The attention to composite fields (see [2] for an overview) stems from the fact that the effective action for composite fields suggested in [1] has been applied to quantum field models such as [6-8], including the Early Universe, Inflationary Universe, Standard Model and SUSY theories [9-13]. The study of BRST-invariant renormalizability in Yang-Mills theories, which includes $N=1$ SUSY formulations [14-16], the functional renormalization group [17-21] and the Gribov horizon [22-24], appears to be promising within the concept of soft BRST symmetry breaking [25-28] and the local composite operator technique [29,30] as applied to arbitrary backgrounds [31]. In its turn, the background field method [3-5] presents the quantization of Yang-Mills theories using background gauges $[5,32,33]$ in such a way that provides an invariance of the effective action under the gauge transformations of background fields and reproduces physical results with essential simplifications in the Feynman diagrams, thereby providing insight into diverse quantum properties of gauge theories [34-43]; for recent developments, see [44-48].

The present article is devoted to quantum non-Abelian gauge models with composite and background fields, whose consistent analysis isfocused on Yang-Mills theories quantized using the Faddeev-Popov method [49] in a combined presence of composite and background fields. A joint treatment of Yang-Mills fields $A_{\mu}$ with composite and background ones requires a systematic consideration of these ingredients as interrelated. We forward the symmetry principle as such a systematic concept. In fact, suppose that a generating functional $Z(J, L)$ of Green's functions with 
composite fields is given, depending on sources $J_{A}$ for the quantum fields $\phi^{A}$, as well as on sources $L_{m}$ for the composite fields $\sigma^{m}(\phi)$. A question then naturally arises of how one can introduce some background fields $B_{\mu}$ in a way that leads to an extended functional $Z(B, J, L)$ reflecting the possible symmetries of $Z(J, L)$. Let us next suppose that a generating functional $Z(B, J)$ of Green's functions, also with certain symmetries, is given in the background field method, and then the question is how some composite fields $\sigma^{m}(\phi, B)$ with sources $L_{m}$ can be introduced for the resulting $Z(B, J, L)$ to inherit the given symmetries. It turns out that these two approaches are equivalent in the following sense.

According to the first approach, a generating functional $Z(J, L)$ is given,

$$
Z(J, L)=\int d \phi \exp \left\{\frac{i}{\hbar}\left[S_{\mathrm{FP}}(\phi)+J_{A} \phi^{A}+L_{m} \sigma^{m}(\phi)\right]\right\},
$$

corresponding to the Faddeev-Popov action $S_{\mathrm{FP}}(\phi)$ of a Yang-Mills theory with composite fields $\sigma^{m}(\phi)$. A background field $B_{\mu}$ can then be introduced by localizing the inherent global symmetry of $Z(J, L)$ under $S U(N)$ transformations (rotations for $J_{A}$ and tensor transformations for $L_{m}$ ) in such a way that $Z(B, J, L)$ defined as

$$
Z(B, J, L)=\left.Z(J, L)\right|_{\partial_{\mu} \rightarrow D_{\mu}(B)}
$$

is invariant under local $S U(N)$ transformations of the sources $J_{A}, L_{m}$, accompanied by gauge transformations of the field $B_{\mu}$ with an associated covariant derivative $D_{\mu}(B)$, where the precise meaning of $\partial_{\mu} \rightarrow D_{\mu}(B)$ in (2) is given by (19) of Section 2. The original $S_{\mathrm{FP}}(\phi)$ becomes thereby modified to the Faddeev-Popov action of the background field method, $S_{\mathrm{FP}}(\phi, B)$, which is related to $S_{\mathrm{FP}}(\phi)$ by so-called background and quantum transformations of this method (see Section 2.2).

According to the second approach, a generating functional $Z(B, J)$ is constructed using the background field method for Yang-Mills theories,

$$
Z(B, J)=\int d \phi \exp \left\{\frac{i}{\hbar}\left[S_{\mathrm{FP}}(\phi, B)+J_{A} \phi^{A}\right]\right\}
$$

which implies

$$
S_{\mathrm{FP}}(\phi, B)=\left.S_{\mathrm{FP}}(\phi)\right|_{\partial_{\mu} \rightarrow D_{\mu}(B)} .
$$

Some composite fields $\sigma^{m}(\phi, B)$ with sources $L_{m}$ can then be introduced on condition that the resulting generating functional

$$
Z(B, J, L)=\int d \phi \exp \left\{\frac{i}{\hbar}\left[S_{\mathrm{FP}}(\phi, B)+J_{A} \phi^{A}+L_{m} \sigma^{m}(\phi, B)\right]\right\}
$$

should inherit the symmetry of $Z(B, J)$ under local $S U(N)$ rotations of the sources $J_{A}$ accompanied by gauge transformations of the background field $B_{\mu}$ with the covariant derivative $D_{\mu}(B)$. This symmetry requirement for $Z(B, J, L)$ is satisfied by a local $S U(N)$ tensor transformation law imposed on $\sigma^{m}(\phi, B)$ and is provided by $B_{\mu}$ entering the composite fields $\sigma^{m}(\phi, B)$ by means of the covariant derivative $D_{\mu}(B)$, which implies

$$
\sigma^{m}(\phi, B)=\left.\sigma^{m}(\phi)\right|_{\partial_{\mu} \rightarrow D_{\mu}(B)}
$$

for certain $\sigma^{m}(\phi)$, and thereby we return to the first approach. In the main part of the article, we implement the first approach as a starting point of our systematic analysis, assuming the composite fields to be local, whereas in the remaining part we show how the first and second approaches can be extended beyond the given assumptions by considering the Volovich-Katanaev model of two-dimensional gravity with dynamical torsion [50] and the Gribov-Zwanziger theory [23,24].

Two-dimensional models of gravity [50-61] and supergravity [62-65] are of interest in view of their close relation to string and superstring theory. Simple two-dimensional models also provide a deeper insight into classical and quantum properties of gravity in higher dimensions, while in some 
cases these models are exactly solvable at the classical level. One of the two-dimensional gravity models that has been widely discussed at the classical [66-70] and quantum [71-75] levels is the model [50] suggested in the context of bosonic string theory with dynamical torsion [76] in order to address some problems of string theory. Thus, it has been shown [76], using the path integral approach, that a string with dynamical torsion has no critical dimension. The model [50] presents the most general theory of two-dimensional $R^{2}$-gravity with independent dynamical torsion leading to second-order equations of motion for the zweibein and Lorentz connection. The model also contains solutions with constant curvature and zero torsion, thereby incorporating some other two-dimensional gravity models [51,52,61] whose actions, as compared to that of [50], do not allow a purely geometric interpretation. Being quantized in the background field method, the model yields a gauge-invariant background effective action [75].

In quantum Yang-Mills theories using differential (e.g., Landau or Feynman) gauges, the non-Abelian nature of the gauge group features the Gribov ambiguity [22] implying a residual gauge-invariance due to Gribov copies, which are removed by means of a Gribov horizon [22] implemented in the Gribov-Zwanziger model [23,24] being a quantum Yang-Mills theory in Landau gauge and including an additive horizon functional in terms of a non-local composite field [77]. The issue of bringing the horizon functional to other gauges has been settled due to the concept of finite field-dependent BRST transformations [26,28,78-84], which allows one to present the horizon functional using different gauges in a way consistent with the gauge-independence of the path integral, based on the Gribov-Zwanziger recipe $[23,24]$ and starting from a BRST-invariant Yang-Mills quantum action in Landau gauge. The horizon functional in covariant $R_{\xi}$ gauges has been given by $[26,28,82,85]$ (see also [86,87] for a BRST-invariant horizon) and later extended to the Standard Model in [88,89].

The article is organized as follows. In Section 2, a generating functional of Green's functions with composite and background fields in Yang-Mills theories is introduced, as well as a generating functional of vertex Green's functions (effective action). Analyzing the dependence of the generating functionals of Green's functions upon a choice of gauge-fixing, we find a gauge variation of the effective action in terms of a nilpotent operator depending on the composite fields and determine the conditions of on-shell gauge-independence. Besides, the effective action $\Gamma_{\text {eff }}(B, \Sigma)$, with a background field $B_{\mu}$ and a set of auxiliary tensor fields $\Sigma^{m}$ associated with $\sigma^{m}$, is found to exhibit a local symmetry under the gauge transformations of $B_{\mu}$ combined with the local $S U(N)$ transformations of $\Sigma^{m}$. In Section 3, we examine the Volovich-Katanaev model [50] quantized according to the background field method in [75]. As an extension of our second approach (3)-(5) beyond the Yang-Mills case, the quantized two-dimensional gravity [75] is modified by the presence of local composite fields, and the corresponding background effective action is found to be gauge-invariant in a way similar to the Yang-Mills case. In Section 4, we modify the Gribov-Zwanziger model [23,24] by introducing a background field, which extends our first approach (1), (2) beyond the Yang-Mills case with local composite fields and thereby yields a gauge-invariant background effective action. Section 5 is devoted to concluding remarks.

We use DeWitt's condensed notation [90]. The Grassmann parity and ghost number of a quantity $F$ are denoted by $\epsilon(F)$, gh $(F)$, respectively. The supercommutator $[F, G\}$ of any quantities $F, G$ with definite Grassmann parities is given by $[F, G\}=F G-(-1)^{\epsilon(F) \epsilon(G)} G F$. Unless specified by an arrow, derivatives with respect to fields and sources are regarded as left-hand ones.

\section{Generating Functionals and Their Properties}

Let us examine a generating functional $Z(J, L)$ corresponding to the Faddeev-Popov action $S_{\mathrm{FP}}(\phi)$ of a Yang-Mills theory with local composite fields,

$$
Z(J, L)=\int d \phi \exp \left\{\frac{i}{\hbar}\left[S_{\mathrm{FP}}(\phi)+J_{A} \phi^{A}+L_{m} \sigma^{m}(\phi)\right]\right\},
$$


where $L_{m}$ are sources to the composite fields $\sigma^{m}(\phi)$,

$$
\sigma^{m}(\phi)=\sum_{n=2} \frac{1}{n !} \Lambda_{A_{1} \ldots A_{n}}^{m} \phi^{A_{n}} \ldots \phi^{A_{1}}
$$

and $J_{A}$ are sources to the fields $\phi^{A}=\left(A^{i}, b^{\alpha}, \bar{c}^{\alpha}, c^{\alpha}\right)$ composed by gauge fields $A^{i}$, (anti)ghost fields $\bar{c}^{\alpha}, c^{\alpha}$, and Nakanishi-Lautrup fields $b^{\alpha}$, with the following distribution of Grassmann parity and ghost number:

$$
\epsilon\left(\phi^{A}\right)=(0,0,1,1), \operatorname{gh}\left(\phi^{A}\right)=(0,0,-1,1), \epsilon\left(J_{A}, L_{m}\right)=\epsilon\left(\phi^{A}, \sigma^{m}\right), \operatorname{gh}\left(J_{A}, L_{m}\right)=-\operatorname{gh}\left(\phi^{A}, \sigma^{m}\right) .
$$

The Faddeev-Popov action $S_{\mathrm{FP}}(\phi)$,

$$
S_{\mathrm{FP}}(\phi)=S_{0}(A)+\Psi(\phi) \overleftarrow{s}
$$

is given in terms of a gauge-invariant classical action $S_{0}(A)$, invariant, $\delta_{\xi} S_{0}(A)=0$, under infinitesimal gauge transformations $\delta_{\xi} A^{i}=R_{\alpha}^{i}(A) \xi^{\alpha}$ with a closed algebra of gauge generators $R_{\alpha}^{i}(A)$,

$$
R_{\alpha, j}^{i}(A) R_{\beta}^{j}(A)-R_{\beta, j}^{i}(A) R_{\alpha}^{j}(A)=F_{\alpha \beta}^{\gamma} R_{\gamma}^{i}(A), \quad F_{\alpha \beta}^{\gamma}=\text { const, } R_{\alpha, j}^{i} \equiv R_{\alpha}^{i} \frac{\overleftarrow{\delta}}{\delta A^{j}}
$$

and a nilpotent Slavnov variation $\overleftarrow{s}$ applied to a gauge Fermion $\Psi(\phi), \epsilon(\Psi)=1$

$$
S_{\mathrm{FP}}(\phi)=S_{0}(A)+\Psi(\phi) \overleftarrow{s}, \quad \Psi(\phi)=\bar{c}^{\alpha} \chi_{\alpha}(\phi), \overleftarrow{s}^{2}=0
$$

where

$$
\phi^{A \overleftarrow{s}}=\left(R_{\alpha}^{i}(A) c^{\alpha}, 0, b^{\alpha}, 1 / 2 F_{\beta \gamma}^{\alpha} c^{\gamma} c^{\beta}\right)
$$

For the explicit field content

$$
\begin{aligned}
i & =(x, p, \mu), \quad \alpha=(x, p), \quad \mu=0, \ldots, D-1, \quad p=1, \ldots, N^{2}-1, \\
\phi^{A} & =\left(A^{p \mid \mu}, b^{p}, \bar{c}^{p}, c^{p}\right), \quad\left(A^{\mu}, b, \bar{c}, c\right) \equiv T^{p}\left(A^{p \mid \mu}, b^{p}, \bar{c}^{p}, c^{p}\right), \quad\left[T^{p}, T^{q}\right]=f^{p q r} T^{r},
\end{aligned}
$$

the field variations $\phi^{A \overleftarrow{s}}$ have the form

$$
\left(A_{\mu}, b, \bar{c}, c\right) \overleftarrow{s}=\left(\left[D_{\mu}(A), c\right], 0, b, g / 2[c, c]_{+}\right),\left(A_{\mu}^{p}, b^{p}, \bar{c}^{p}, c^{p}\right) \overleftarrow{s}=\left(D_{\mu}^{p q}(A) c^{q}, 0, b^{p}, g / 2 f^{p q r} c^{q} c^{r}\right)
$$

where

$$
D_{\mu}(A) \equiv \partial_{\mu}+g A_{\mu}, \quad D_{\mu}^{p q}(A)=\delta^{p q} \partial_{\mu}+g f^{p r q} A_{\mu}^{r},
$$

The classical action $S_{0}(A)$ has the form (in the adjoint representation with Hermitian $T^{p}$ ),

$$
\begin{aligned}
S_{0}(A) & =\frac{1}{2 g^{2}} \int d^{D} x \operatorname{Tr}\left(F_{\mu v} F^{\mu v}\right)=\frac{1}{4} \int d^{D} x F_{\mu v}^{p} F^{p \mid \mu v}, \operatorname{Tr}\left(T^{p} T^{q}\right)=\frac{1}{2} \delta^{p q}, \\
F_{\mu v} & \equiv\left[D_{\mu}(A), D_{v}(A)\right], \quad F_{\mu v}^{p}=\partial_{\mu} A_{v}^{p}-\partial_{v} A_{\mu}^{p}+g f^{p r s} A_{\mu}^{r} A_{v}^{s},
\end{aligned}
$$

and the gauge Fermion $\Psi(\phi)=\bar{c}^{\alpha} \chi_{\alpha}(\phi)$ with some gauge-fixing functions $\chi_{\alpha}(\phi)=\chi^{p}(\phi(x))$ reads

$$
\Psi(\phi)=\int d^{D} x \bar{c}^{p} \chi^{p}(\phi)=2 \int d^{D} x \operatorname{Tr}[\bar{c} \chi(\phi)], \quad \chi(\phi)=T^{p} \chi^{p}(\phi) .
$$


The Faddeev-Popov action $S_{\mathrm{FP}}(\phi)$ in (8), (9) is invariant under two kinds of global transformations: BRST transformations [91-93], $\delta_{\lambda} \phi^{A}=\phi^{A} \overleftarrow{s} \lambda$, with an anticommuting parameter $\lambda$, $\epsilon(\lambda)=1$, and $S U(N)$ rotations (finite $\phi^{A} \stackrel{U}{\rightarrow} \phi^{\prime A}$ and infinitesimal $\delta_{\zeta} \phi^{A}$ ) with even parameters $\varsigma^{p}$,

$$
\begin{aligned}
\left(A_{\mu}, b, \bar{c}, c\right) \stackrel{U}{\rightarrow}\left(A_{\mu}, b, \bar{c}, c\right)^{\prime} & =U\left(A_{\mu}, b, \bar{c}, c\right) U^{-1}, \quad U=\exp \left(-g T^{p} \varsigma^{p}\right), \quad \varsigma^{p}=\text { const }, \\
\delta_{\zeta}\left(A_{\mu}^{p}, b^{p}, \bar{c}^{p}, c^{p}\right) & =g f^{p r q}\left(A_{\mu}^{r}, b^{r}, \bar{c}^{r}, c^{r}\right) \varsigma^{q},
\end{aligned}
$$

or, in a tensor form, via the adjoint representation with a matrix $M^{p q}(\varsigma)$,

$$
\left(A_{\mu}^{p}, b^{p}, \bar{c}^{p}, c^{p}\right)^{\prime}=M^{p q}(\varsigma)\left(A_{\mu}^{q}, b^{q}, \bar{c}^{q}, c^{q}\right), \quad M^{p q}(\varsigma)=\delta^{p q}+g f^{p q r} \varsigma^{r}+O\left(\varsigma^{2}\right) .
$$

The classical action $S_{0}(A)$ in $S_{\mathrm{FP}}(\phi)=S_{0}(A)+\Psi(\phi) \overleftarrow{s}$ is invariant under $A_{\mu} \stackrel{u}{\rightarrow} A_{\mu}^{\prime}$ as a particular case $\left(\xi^{p}(x)=\right.$ const) of invariance under the finite form $A_{\mu} \stackrel{V}{\rightarrow} A_{\mu}^{\prime}$ of gauge transformations

$$
A_{\mu}^{\prime}=V A_{\mu} V^{-1}+g^{-1} V\left(\partial_{\mu} V^{-1}\right), \quad D_{\mu}\left(A^{\prime}\right)=V D_{\mu}(A) V^{-1}, \quad V=\exp \left(-g T^{p} \xi^{p}\right), \quad \xi^{p}=\xi^{p}(x),
$$

while the invariance of $\Psi(\phi) \overleftarrow{s}$ under $\phi^{A} \stackrel{U}{\rightarrow} \phi^{\prime A}$ reflects the explicit form of $\overleftarrow{s}$ and the fact that the gauge functions $\chi^{p}(\phi)$ are local and constructed from the fields $\phi^{A}$, structure constants $f^{p q r}$ and derivatives $\partial_{\mu}$, for instance, in Landau and Feynman gauges,

$$
\chi_{\mathrm{L}}^{p}(\phi)=\partial^{\mu} A_{\mu}^{p}, \quad \chi_{\mathrm{F}}^{p}(\phi)=b^{p}+\partial^{\mu} A_{\mu}^{p},
$$

so that, in particular, $\chi^{p}(\phi)$ transform as $S U(N)$ vectors, with $\Psi(\phi)$ being invariant under $\phi^{A} \stackrel{U}{\rightarrow} \phi^{\prime A}$,

$$
\chi\left(\phi^{\prime}\right)=U \chi(\phi) U^{-1}, \quad \delta \chi^{p}(\phi)=g f^{p r q} \chi^{r}(\phi) \varsigma^{q}
$$

Due to the same reason, local composite fields $\sigma^{m}(\phi)$ constructed from the fields $\phi^{A}$, structure constants $f^{p q r}$ and derivatives $\partial_{\mu}$,

$$
\sigma^{m}(\phi)=\sigma^{p_{1} \cdots p_{k} \mid \mu_{1} \cdots \mu_{l}}(\phi(x)), \quad m=\left(x, p_{1} \cdots p_{k}, \mu_{1} \cdots \mu_{l}\right),
$$

in the path integral (6) for $Z(J, L)$ transform under $\phi^{A} \stackrel{U}{\rightarrow} \phi^{\prime A}$ as tensors with respect to the indices $p_{1}, \ldots, p_{k}$

$$
\begin{aligned}
\sigma^{p_{1} \cdots p_{k} \mid \mu_{1} \cdots \mu_{l}} & =M^{p_{1} q_{1}} \cdots M^{p_{k} q_{k}} \sigma^{q_{1} \cdots q_{k} \mid \mu_{1} \cdots \mu_{l}}, \\
\delta_{\zeta} \sigma^{p_{1} \cdots p_{k} \mid \mu_{1} \cdots \mu_{l}} & =g \sum_{r_{s} \in\left\{r_{1}, \cdots, r_{k}\right\}} f^{p_{s} r_{s} q} \sigma^{p_{1} \cdots r_{s} \cdots p_{k} \mid \mu_{1} \cdots \mu_{l}} \varsigma^{q} \equiv g f^{\{p\} \hat{r} q} \sigma^{p_{1} \cdots \hat{r} \cdots p_{k} \mid \mu_{1} \cdots \mu_{l}} \varsigma^{q},
\end{aligned}
$$

which generalizes the vector transformation. As a result, the exponential in the path integral (6) for $Z(J, L)$ is invariant under $\phi^{A} \stackrel{U}{\rightarrow} \phi^{\prime A}$, along with some global transformations of the sources $J_{A}, L_{m}$,

$$
\left(J_{A}, L_{m}\right) \stackrel{U}{\rightarrow}\left(J_{A}, L_{m}\right)^{\prime}, \quad J_{A}=\left(J_{(A) \mu^{\prime}}^{p} J_{(b)}^{p}, J_{(\bar{c})^{\prime}}^{p}, J_{(c)}^{p}\right), \quad L_{m}=L_{\mu_{1} \cdots \mu_{l}}^{p_{1} \cdots p_{k}},
$$

in a tensor and infinitesimal form,

$$
\begin{aligned}
& \left(J_{(A)^{\prime}}^{p \mid \mu} J_{(b)}^{p}, J_{(\bar{c})^{\prime}}^{p} J_{(c)}^{p}\right)^{\prime}=M^{p q}\left(J_{(A)^{\prime}}^{q \mid \mu} J_{(b)}^{q} J_{(\bar{c})^{\prime}}^{q} J_{(c)}^{q}\right), \quad L_{\mu_{1} \cdots \mu_{l}}^{\prime p_{1} \cdots p_{k}}=M^{p_{1} q_{1}} \cdots M^{p_{k} q_{k}} L_{\mu_{1} \cdots \mu_{l}}^{q_{1} \cdots q_{k}}, \\
& \delta_{\zeta}\left(J_{(A)}^{p \mid \mu}, J_{(b)}^{p}, J_{(\bar{c})}^{p}, J_{(c)}^{p}\right)=g f^{p r q}\left(J_{(A)}^{r \mid \mu}, J_{(b)}^{r}, J_{(\bar{c})}^{r} J_{(c)}^{r}\right) \zeta^{q}, \quad \delta_{\zeta} L_{\mu_{1} \cdots \mu_{l}}^{p_{1} \cdots p_{k}}=g f^{\{p\} r q} L_{\mu_{1} \cdots \mu_{l}}^{p_{1} \cdots \hat{r} \cdots p_{k}} S^{q},
\end{aligned}
$$

which entails the invariance of the source term $J_{A} \phi^{A}+L_{m} \sigma^{m}(\phi)$. 
Let us introduce an additional field $B_{\mu}=B_{\mu}^{p} T^{p}$ having a gauge transformation as in (11),

$$
B_{\mu} \stackrel{V}{\rightarrow} B_{\mu}^{\prime}=V B_{\mu} V^{-1}+g^{-1} V\left(\partial_{\mu} V^{-1}\right)
$$

with the inherent property

$$
D_{\mu}\left(B^{\prime}\right)=V D_{\mu}(B) V^{-1}, \quad D_{\mu}(B) \equiv \partial_{\mu}+g B_{\mu},
$$

and subject the exponential in the path integral (6) to the modification

$$
\left.\exp \left\{\frac{i}{\hbar}\left[S_{\mathrm{FP}}(\phi)+J \phi+L \sigma(\phi)\right]\right\}\right|_{\partial_{\mu} \rightarrow D_{\mu}(B)} \equiv \exp \left\{\frac{i}{\hbar}\left[S_{\mathrm{FP}}(\phi, B)+J \phi+L \sigma(\phi, B)\right]\right\},
$$

where the replacement $\partial_{\mu} \rightarrow D_{\mu}(B)$ reads as

$$
\partial_{\mu} \rightarrow D_{\mu}(B):\left[\partial_{\mu}, \bullet\right] \rightarrow\left[D_{\mu}(B), \bullet\right] \Rightarrow\left[D_{\mu}(A), \bullet\right] \rightarrow\left[D_{\mu}(A+B), \bullet\right],
$$

so that

$$
F_{\mu v}(A) \rightarrow\left[D_{\mu}(A+B), D_{v}(A+B)\right]=F_{\mu \nu}(A+B) .
$$

Due to the transformation property (17) of the derivative $D_{\mu}(B)$, the generating functional $Z(B, J, L)$ modified by the field $B_{\mu}$ according to (18), (19),

$$
Z(B, J, L)=\int d \phi \exp \left\{\frac{i}{\hbar}\left[S_{\mathrm{FP}}(\phi, B)+J_{A} \phi^{A}+L_{m} \sigma^{m}(\phi, B)\right]\right\},
$$

is invariant under a set of local transformations,

$$
\begin{aligned}
\delta_{\xi} B_{\mu}^{p} & =D_{\mu}^{p q}(B) \xi^{q}, \\
\delta_{\xi}\left(J_{(A)}^{p \mid \mu}, J_{(b)}^{p}, J_{(\bar{c},}^{p} J_{(c)}^{p}\right) & =g f^{p r q}\left(J_{(A)}^{r \mid \mu}, J_{(b)}^{r}, J_{(\bar{c})}^{r}, J_{(c)}^{r}\right) \xi^{q}, \\
\delta_{\xi} L_{\mu_{1} \cdots \mu_{l}}^{p_{1} \cdots p_{k}} & =g f^{\{p\} \hat{r} q} L_{\mu_{1} \cdots \mu_{l}}^{p_{1} \cdots \hat{r} \cdots p_{k}} \xi^{q},
\end{aligned}
$$

given by the gauge transformations (16) of the field $B_{\mu}$ combined with a localized form $U(\varsigma) \rightarrow V(\xi)$ of the transformations (14), (15) for the sources $J_{A}, L_{m}$ with infinitesimal parameters $\xi^{p}$,

$$
\left(B_{\mu}, J_{A}, L_{m}\right) \stackrel{V}{\rightarrow}\left(B_{\mu}, J_{A}, L_{m}\right)^{\prime} .
$$

The invariance property $Z\left(B^{\prime}, J^{\prime}, L^{\prime}\right)=Z(B, J, L)$ can be established by applying to the transformed path integral $Z\left(B^{\prime}, J^{\prime}, L^{\prime}\right)$ a compensating change of the integration variables:

$$
\delta_{\xi}\left(A_{\mu}^{p}, b^{p}, \bar{c}^{p}, c^{p}\right)=g f^{p r q}\left(A_{\mu}^{r}, b^{r}, \bar{c}^{r}, c^{r}\right) \xi^{q}, \quad\left(A_{\mu}, b, \bar{c}, c\right) \stackrel{V}{\rightarrow}\left(A_{\mu}, b, \bar{c}, c\right)^{\prime},
$$

whose Jacobian equals to unity in view of the complete antisymmetry of the structure constants. The invariance of $Z(B, J, L)$ can be recast in the form

$$
\begin{aligned}
& \int d^{D} x\left\{\left[D_{\mu}^{p q}(B) \xi^{q}\right] \frac{\vec{\delta}}{\delta B_{\mu}^{p}}+g \xi^{q} f^{\{p\} \hat{r q}} L_{\mu_{1} \cdots \mu_{l}}^{p_{1} \cdots \hat{p_{k} \cdots p_{k}}} \frac{\vec{\delta}}{\delta L_{\mu_{1} \cdots \cdots \mu_{l}}^{p_{1} \cdots p_{k}}}\right. \\
& \left.+g \xi^{q} f^{p r q}\left(J_{(A)}^{r \mid \mu} \frac{\vec{\delta}}{\delta J_{(A)}^{p \mid \mu}}+J_{(b)}^{r} \frac{\vec{\delta}}{\delta J_{(b)}^{p}}+J_{(\bar{c})}^{r} \frac{\vec{\delta}}{\delta J_{(\bar{c})}^{p}}+J_{(c)}^{r} \frac{\vec{\delta}}{\delta J_{(c)}^{p}}\right)\right\} Z(B, J, L)=0 .
\end{aligned}
$$




\subsection{Effective Action and Gauge Dependence}

Let us present a generating functional of vertex Green's functions and examine its gaugedependence properties. To do so, we first introduce an extended generating functional $Z\left(B, J, L, \phi^{*}\right)$,

$$
Z\left(B, J, L, \phi^{*}\right)=\int d \phi \exp \left\{\frac{i}{\hbar}\left[S_{\text {ext }}\left(\phi, \phi^{*}, B\right)+J_{A} \phi^{A}+L_{m} \sigma^{m}(\phi, B)\right]\right\},
$$

with an extended quantum action $S_{\text {ext }}\left(\phi, \phi^{*}, B\right)$ defined as

$$
S_{\text {ext }}\left(\phi, \phi^{*}, B\right)=S_{\mathrm{FP}}(\phi, B)+\phi_{A}^{*}\left(\phi^{A} \overleftarrow{s}_{\mathrm{q}}\right), \quad \overleftarrow{s}_{\mathrm{q}}=\overleftarrow{s}_{\partial_{\mu} \rightarrow D_{\mu}(B)}, \quad \overleftarrow{s}_{\mathrm{q}} \overleftarrow{s}_{\mathrm{q}}=0
$$

where $\phi_{A}^{*}$ is a set of antifields introduced as sources to the variations $\phi^{A} \overleftarrow{S}_{\mathrm{q}}$. Given the properties $S_{\mathrm{FP}}(\phi) \overleftarrow{s}=0$ and $\overleftarrow{s}_{\mathrm{q}} \overleftarrow{s}_{\mathrm{q}}=0$, the extended quantum action $S_{\text {ext }}\left(\phi, \phi^{*}, B\right)$ satisfies the identity

$$
S_{\text {ext }}\left(\phi, \phi^{*}, B\right) \overleftarrow{s}_{\mathrm{q}}=S_{\mathrm{FP}}(\phi, B) \overleftarrow{s}_{\mathrm{q}}=S_{\mathrm{FP}}(\phi) \overleftarrow{s}_{\partial_{\mu} \rightarrow D_{\mu}(B)}=0
$$

having an equivalent form, implied by the antisymmetry of the structure constants:

$$
\Delta \exp (i / \hbar) S_{\mathrm{ext}}=0, \quad \Delta \equiv(-1)^{\epsilon\left(\phi^{A}\right)} \frac{\vec{\delta}}{\delta \phi^{A}} \frac{\vec{\delta}}{\delta \phi_{A}^{*}}, \quad \Delta^{2}=0 .
$$

In view of (24), (25), the variation $\delta_{\Psi} Z\left(B, J, L, \phi^{*}\right)$ related to a change $\delta \Psi(\phi, B)$ has the form

$$
\begin{aligned}
& \delta_{\Psi} Z\left(B, J, L, \phi^{*}\right)=\int d \phi \exp \left\{\frac{i}{\hbar}\left[J_{A} \phi^{A}+L_{m} \sigma^{m}(\phi, B)\right]\right\} \delta_{\Psi} \exp \left[\frac{i}{\hbar} S_{\text {ext }}\left(\phi, \phi^{*}, B\right)\right] \\
& \delta_{\Psi} \exp \left(\frac{i}{\hbar} S_{\text {ext }}\right)=\frac{i}{\hbar} \delta \Psi \overleftarrow{s}_{\mathrm{q}} \exp \left(\frac{i}{\hbar} S_{\text {ext }}\right)=-\Delta\left[\delta \Psi \exp \left(\frac{i}{\hbar} S_{\text {ext }}\right)\right], \delta \overleftarrow{s}_{\mathrm{q}}=\delta \Psi, A\left(\phi^{A} \overleftarrow{s}_{\mathrm{q}}\right)
\end{aligned}
$$

which can be represented as

$$
\delta_{\Psi} Z=\delta \Psi, A\left(\frac{\hbar}{i} \frac{\vec{\delta}}{\delta J}, B\right) \frac{\vec{\delta}}{\delta \phi_{A}^{*}} \mathrm{Z}=\frac{i}{\hbar} \hat{\omega} \delta \Psi\left(\frac{\hbar}{i} \frac{\vec{\delta}}{\delta J}, B\right) Z,
$$

where the second equality is a result of integration by parts in (26), and $\hat{\omega}$ is a nilpotent operator (which is also found in the Ward identity $\hat{\omega} Z=0$ ), namely,

$$
\hat{\omega}=\left[J_{A}+L_{m} \sigma_{, A}^{m}\left(\frac{\hbar}{i} \frac{\vec{\delta}}{\delta J}, B\right)\right] \frac{\vec{\delta}}{\delta \phi_{A}^{*}}, \hat{\omega}^{2}=0 .
$$

In terms of a generating functional $\Gamma\left(B, \phi, \Sigma, \phi^{*}\right)$ of vertex Green's functions with composite fields (on a background $B_{\mu}$ ) given by a double Legendre transformation [94],

$$
\Gamma\left(B, \phi, \Sigma, \phi^{*}\right)=W\left(B, J, L, \phi^{*}\right)-J_{A} \phi^{A}-L_{m}\left[\sigma^{m}(\phi, B)+\Sigma^{m}\right], W=(\hbar / i) \ln Z,
$$

where

$$
\phi^{A}=\frac{\vec{\delta} W}{\delta J_{A}}, \Sigma^{m}=\frac{\vec{\delta} W}{\delta L_{m}}-\sigma^{m}\left(\frac{\vec{\delta} W}{\delta J}, B\right),-J_{A}=\Gamma \frac{\overleftarrow{\delta}}{\delta \phi^{A}}+L_{m} \sigma_{, A}^{m}(\phi, B),-L_{m}=\Gamma \frac{\overleftarrow{\delta}}{\delta \Sigma^{m}}
$$


The variation $\delta_{\Psi} \Gamma\left(B, \phi, \Sigma, \phi^{*}\right)$ induced by (27), with account taken of $\delta_{\Psi} \Gamma=\delta_{\Psi} W$, reads

$$
\begin{aligned}
& \delta_{\Psi} \Gamma=\left\langle\langle\delta \Psi\rangle \frac{\overleftarrow{\delta}}{\delta \phi^{A}} \frac{\vec{\delta} \Gamma}{\delta \phi_{A}^{*}}-\langle\langle\delta \Psi\rangle\rangle \frac{\overleftarrow{\delta}}{\delta \phi_{A}^{*}} \frac{\vec{\delta} \Gamma}{\delta \phi^{A}}+\left[\sigma_{, A}^{m}(\hat{\phi}, B)-\sigma_{, A}^{m}(\phi, B)\right] \frac{\left.\vec{\delta} \Gamma \frac{\vec{\delta}}{\delta \phi_{A}^{*}} \frac{\overrightarrow{\delta \Sigma^{m}}}{\delta \Sigma^{\prime}}\langle\delta \Psi\rangle\right\rangle}{-}+\frac{i}{\hbar}\left(\left[\Gamma \frac{\overleftarrow{\delta}}{\delta \Sigma^{m}}\left(\sigma_{, C}^{m}(\hat{\phi}, B) \frac{\vec{\delta} \Gamma}{\delta \phi_{C}^{*}}\right), \Phi^{\mathrm{a}}\right\} \frac{\vec{\delta}}{\delta \Phi^{\mathrm{a}}}+\left[\Gamma \frac{\overleftarrow{\delta}}{\delta \Sigma^{m}}\left(\sigma_{, C}^{m}(\hat{\phi}, B) \frac{\vec{\delta} \Gamma}{\delta \phi_{C}^{*}}\right), \sigma^{n}(\phi, B)\right\} \frac{\vec{\delta}}{\delta \Sigma^{n}}\right)\langle\langle\delta \Psi\rangle\rangle\right. \\
& +\frac{i}{\hbar}(-1)^{\epsilon\left(\sigma^{n}\right)+\epsilon\left(\phi^{D}\right)} \sigma_{, D}^{n}(\phi, B)\left[\Gamma \frac{\overleftarrow{\delta}}{\delta \Sigma^{m}}\left(\sigma_{, C}^{m}(\hat{\phi}, B) \frac{\vec{\delta} \Gamma}{\delta \phi_{C}^{*}}\right), \phi^{D}\right\} \frac{\vec{\delta}}{\delta \Sigma^{n}}\langle\langle\delta \Psi\rangle\rangle \\
& +(-1)^{\epsilon\left(\sigma^{m}\right)+\epsilon\left(\phi^{D}\right) \epsilon\left(\phi^{A}\right)}\left[\sigma_{, D}^{m}(\phi, B), \Gamma \frac{\overleftarrow{\delta}}{\delta \Sigma^{n}} \sigma_{, A}^{n}(\hat{\phi}, B)\right\}\left(G^{\prime \prime-1}\right)^{A a}\left(\frac{\vec{\delta}}{\delta \Phi^{\mathrm{a}}} \frac{\vec{\delta} \Gamma}{\delta \phi_{D}^{*}}\right) \frac{\vec{\delta}\langle\langle\delta \Psi\rangle\rangle}{\delta \Sigma^{m}} \\
& \delta_{\Psi} \Gamma \equiv \hat{\omega}_{\Gamma}\langle\langle\delta \Psi\rangle\rangle, \quad\langle\langle\delta \Psi\rangle\rangle \equiv \delta \Psi(\hat{\phi}, B),
\end{aligned}
$$

where

$$
\begin{aligned}
\hat{\phi}^{A} & =\phi^{A}+i \hbar\left(G^{\prime \prime-1}\right)^{A a} \frac{\vec{\delta}}{\delta \Phi^{\mathrm{a}}}, \quad \Phi^{\mathrm{a}}=\left(\phi^{A}, \Sigma^{m}\right) \\
G_{\mathrm{ab}}^{\prime \prime} & =\frac{\vec{\delta}}{\delta \Phi^{\mathrm{a}}} F_{\mathrm{b}}, \quad F_{\mathrm{a}}=\left(\Gamma \frac{\overleftarrow{\delta}}{\delta \phi^{A}}-\Gamma \frac{\overleftarrow{\delta}}{\delta \Sigma^{n}} \sigma_{, A}^{n}(\phi, B), \Gamma \frac{\overleftarrow{\delta}}{\delta \Sigma^{m}}\right)
\end{aligned}
$$

In (30), (31), $\hat{\omega}_{\Gamma}$ is a Legendre transform of $\hat{\omega}_{W} \equiv e^{-i / \hbar W} \hat{\omega} e^{i / \hbar W}$, with $\hat{\omega}$ given by (28), and therefore $\hat{\omega}_{\Gamma}$ inherits the nilpotency of $\hat{\omega}$, namely, $\hat{\omega}_{\Gamma}^{2}=\hat{\omega}_{W}^{2}=0$. From (30) it follows, according to $[95,96]$, that the generating functional $\Gamma\left(B, \phi, \Sigma, \phi^{*}\right)$ is gauge-independent, $\delta_{\Psi} \Gamma=0$, on the extremals

$$
\frac{\delta \Gamma}{\delta \phi^{A}}=\frac{\delta \Gamma}{\delta \Sigma^{m}}=0,
$$

so that the effective action $\Gamma_{\text {eff }}=\Gamma_{\text {eff }}(B, \Sigma)$ with composite and background fields defined as

$$
\Gamma_{\text {eff }}(B, \Sigma)=\left.\Gamma\left(B, \phi, \Sigma, \phi^{*}\right)\right|_{\phi=\phi^{*}=0}
$$

is gauge-independent, $\delta_{\Psi} \Gamma_{\text {eff }}=0$, on the extremals (32) restricted to the hypersurface $\phi^{A}=\phi_{A}^{*}=0$ of vanishing quantum fields $\phi^{A}$ and antifields $\phi_{A}^{*}$. This result on gauge dependence, in fact, holds true for general gauge theories, whose gauge algebra may be open, and whose gauge generators may be reducible, given an appropriate field-antifield structure. Besides, since the restricted generating functional $Z(B, J, L)$, as we set $\phi^{*}=0$ in (24), satisfies the identity (23), which is also valid for the related functional $W(B, J, L)$ as we substitute $Z=\exp [(i / \hbar) W]$, the effective action $\Gamma_{\text {eff }}(B, \Sigma)$ defined by (29), (33) obeys an equality resulting from a Legendre transform of (23) written in terms of $W(B, J, L)=\left.W\left(B, J, L, \phi^{*}\right)\right|_{\phi^{*}=0}$ and then reduced to $\phi^{A}=0$,

$$
\int d^{D} x\left\{\left[D_{\mu}^{p q}(B) \xi^{q}\right] \frac{\vec{\delta}}{\delta B_{\mu}^{p}}+g f^{\{p\} \hat{r} q} \sum_{\mu_{1} \cdots \mu_{l}}^{p_{1} \cdots \hat{r} \cdots p_{k}} \xi^{q} \frac{\vec{\delta}}{\delta \sum_{\mu_{1} \cdots \mu_{l}}^{p_{1} \cdots p_{k}}}\right\} \Gamma_{\text {eff }}(B, \Sigma)=0,
$$

as a consequence of the following identity implied by the notation $f^{\{p\} \hat{r} q}$ in (13), due to the antisymmetry of the structure constants:

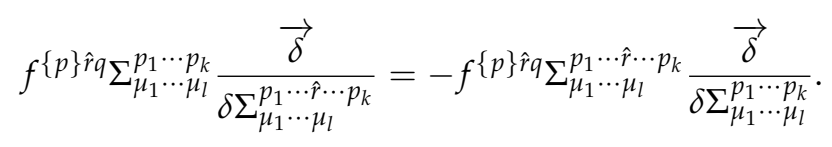

The effective action is thereby invariant, $\delta_{\xi} \Gamma_{\text {eff }}=0$, under the local transformations

$$
\delta_{\xi} B_{\mu}^{p}=D_{\mu}^{p q}(B) \xi^{q}, \quad \delta_{\xi} \sum_{\mu_{1} \cdots \mu_{l}}^{p_{1} \cdots p_{k}}=g f^{\{p\} \hat{r} q} \sum_{\mu_{1} \cdots \mu_{l}}^{p_{1} \cdots \hat{r} \cdots p_{k}} \xi^{q},
$$


which consist of the initial gauge transformations for the background field $B_{\mu}^{p}$ and of the local $S U(N)$ transformations for the fields $\Sigma_{\mu_{1} \cdots \mu_{j}}^{p_{1} \cdots p_{i}}$. Note that we assume the existence of a "deep" gauge-invariant regularization preserving the Ward identities (see, e.g., [14]), and expect the corresponding renormalized generating functionals to obey the same properties as the unrenormalized ones.

\subsection{Background Field Interpretation}

In order to interpret the generating functional $Z(B, J, L)$ in (21), note that the modified Faddeev-Popov action $S_{\mathrm{FP}}(\phi, B)$ defined as (18)-(20) is invariant under the finite local transformations

$$
\left(A_{\mu}, b, \bar{c}, c\right) \stackrel{V}{\rightarrow} V\left(A_{\mu}, b, \bar{c}, c\right) V^{-1}, \quad B_{\mu} \stackrel{V}{\rightarrow} V B_{\mu} V^{-1}+g^{-1} V \partial_{\mu} V^{-1}
$$

and acquires the form

$$
S_{\mathrm{FP}}(\phi, B)=S_{0}(A+B)+\Psi(\phi, B) \overleftarrow{s}_{\mathrm{q}}
$$

where

$$
\begin{aligned}
S_{0}(A+B) & =\left.S_{0}(A)\right|_{\left[\partial_{\mu}, \bullet\right] \rightarrow\left[D_{\mu}(B), \bullet\right]}=\left.S_{0}(A)\right|_{D_{\mu}(A) \rightarrow D_{\mu}(A+B)}, \\
\Psi(\phi, B) & =\left.\Psi(\phi)\right|_{\left[\partial_{\mu}, \bullet\right] \rightarrow\left[D_{\mu}(B), \bullet\right]}, \quad \overleftarrow{S}_{\mathrm{q}}=\left.\overleftarrow{s}\right|_{D_{\mu}(A) \rightarrow D_{\mu}(A+B)},
\end{aligned}
$$

and the variation $\overleftarrow{s}_{\text {q }}$ reads explicitly

$$
\begin{aligned}
\left(A_{\mu}, b, \bar{c}, c\right) \overleftarrow{s}_{\mathrm{q}} & =\left(\left[D_{\mu}(A+B), c\right], 0, b, g / 2[c, c]_{+}\right) \\
\left(A_{\mu}^{p}, b^{p}, \bar{c}^{p}, c^{p}\right) \overleftarrow{s}_{\mathrm{q}} & =\left(D_{\mu}^{p q}(A+B) c^{q}, 0, b^{p}, g / 2 f^{p q r} c^{q} c^{r}\right) .
\end{aligned}
$$

Thus, the Landau and Feynman gauges (12) are modified to the respective background gauges

$$
\chi_{\mathrm{L}}^{p}(\phi, B)=D_{\mu}^{p q}(B) A^{q \mid \mu}, \quad \chi_{\mathrm{F}}^{p}(\phi, B)=b^{p}+D_{\mu}^{p q}(B) A^{q \mid \mu} .
$$

In the background field method, a quantum action $S_{\mathrm{FP}}(\phi, B)$ given by (38), (40) is known as the Faddeev-Popov action with a background field $B_{\mu}$. The quantum action $S_{\mathrm{FP}}(\phi, B)$ is invariant under global transformations of $\phi^{A}$, with a nilpotent generator $\overleftarrow{s}_{\mathrm{q}}$ and an anticommuting parameter $\lambda$ :

$$
\delta_{\lambda} S_{\mathrm{FP}}(\phi, B)=0, \quad \delta \phi^{A}=\phi^{A} \overleftarrow{s}_{\mathrm{q}} \lambda, \quad \epsilon(\lambda)=1
$$

Infinitesimally, the local transformations (37) for the fields $A_{\mu}, B_{\mu}$ are known as background transformations, $\delta_{\mathrm{b}}\left(A_{\mu}, B_{\mu}\right)$, and the transformations of $A_{\mu}, B_{\mu}$ corresponding to the modified Slavnov variation $\overleftarrow{s}_{\mathrm{q}}$ in (38), (40), (42) are known as quantum transformations, $\delta_{\mathrm{q}}\left(A_{\mu}, B_{\mu}\right)$,

$$
\begin{array}{ll}
\delta_{\mathrm{b}} A_{\mu}=g\left[A_{\mu}, T^{p} \xi^{p}\right], & \delta_{\mathrm{b}} B_{\mu}=\left[D_{\mu}(B), T^{p} \xi^{p}\right], \\
\delta_{\mathrm{q}} A_{\mu}=\left[D_{\mu}(A+B), T^{p} \xi^{p}\right], & \delta_{\mathrm{q}} B_{\mu}=0,
\end{array}
$$

while the classical action $S_{0}(A+B)$ is invariant under both types of such transformations. In this regard, the family of background gauges $\chi^{p}(\phi, B)=\tilde{\chi}^{p}(A, B)+(\alpha / 2) b^{p}$, parameterized by $\alpha \neq 0$ and defined as (39),

$$
\chi^{p}(A, B)=\left.\chi^{p}(A)\right|_{\left[\partial_{\mu}, \bullet\right] \rightarrow\left[D_{\mu}(B), \bullet\right]},
$$


with the Nakanishi-Lautrup fields $b^{p}$ integrated out of (21) by the shift $b^{p} \rightarrow b^{p}+\alpha^{-1} \tilde{\chi}$ at the vanishing sources, $J=L=0$, transforms the vacuum functional $Z(B)$ to the representation (for the convenience of Section 3, we denote $A \equiv Q$ ),

$$
\begin{aligned}
Z(B) & =\int d Q d \bar{c} d c \exp \left\{\frac{i}{\hbar}\left[S_{0}(Q+B)+S_{\mathrm{gf}}(Q, B)+S_{\mathrm{gh}}(Q, B ; \bar{c}, c)\right]\right\}, \\
S_{\mathrm{gf}}(Q, B) & =-\frac{1}{2 \alpha} \int d^{D} x \tilde{\chi}^{p} \tilde{\chi}^{p}, \quad S_{\mathrm{gh}}(Q, B)=\left.\int d^{D} x \bar{c}^{p} \delta_{\mathrm{q}} \tilde{\chi}^{p}\right|_{\xi \rightarrow c},
\end{aligned}
$$

where the gauge-fixing term $S_{\mathrm{gf}}=S_{\mathrm{gf}}(Q, B)$ is invariant, $\delta_{\mathrm{b}} S_{\mathrm{gf}}=0$ under the background transformations, due to $\delta_{\mathrm{b}} \tilde{\chi}^{p}=g f^{p r q} \tilde{\chi}^{r} \xi^{q}$, which can be employed as a definition for the quantum action in background gauges $\tilde{\chi}^{p}(Q, B)$ depending on the quantum and background fields, with the related background and quantum transformations (see also [5]),

$$
\begin{array}{ll}
\delta_{\mathrm{b}} B_{\mu}^{p}=D_{\mu}^{p q}(B) \xi^{q}, & \delta_{\mathrm{b}} Q_{\mu}^{p}=g f^{p r q} Q_{\mu}^{r} \xi^{q}, \\
\delta_{\mathrm{q}} B_{\mu}^{p}=0, & \delta_{\mathrm{q}} Q_{\mu}^{p}=D_{\mu}^{p q}(Q+B) \xi^{q} .
\end{array}
$$

The quantum action and the integrand of $Z(B)$ in (43) are invariant under the residual local transformations (37),

$$
\left(Q_{\mu}, \bar{c}, c\right) \stackrel{V}{\rightarrow} V\left(Q_{\mu}, \bar{c}, c\right) V^{-1}, \quad B_{\mu} \stackrel{V}{\rightarrow} V B_{\mu} V^{-1}+g^{-1} V \partial_{\mu} V^{-1},
$$

corresponding, at the infinitesimal level $\delta_{\mathcal{\xi}}\left(B_{\mu}, Q_{\mu}, \bar{c}, c\right)$, to the background transformations $\delta_{\mathrm{b}}$ along with some compensating local transformations of the ghost fields:

$$
\delta_{\xi}\left(B_{\mu}^{p}, Q_{\mu}^{p}, \bar{c}^{p}, c^{p}\right)=\left(\delta_{\mathrm{b}} B_{\mu}^{p}, \delta_{\mathrm{b}} Q_{\mu}^{p}, g f^{p r q} \bar{c}^{r} \xi^{q}, g f^{p r q} c^{r} \xi^{q}\right) .
$$

In view of the above, we interpret $Z(B, J, L)$ in (6), (18), (19), (21) as a generating functional of Green's functions for Yang-Mills theories with composite fields in the background field method, or as a generating functional of Green's functions with composite and background fields in such theories. As has been shown, this interpretation provides for such theories the existence of a corresponding gauge-invariant (36) effective action, being gauge-independent on the extremals (32) for the extended generating functional of vertex functions (29) when these extremals are restricted to the hypersurface of vanishing antifields and quantum fields.

\section{Volovich-Katanaev Model}

Let us examine the model of two-dimensional gravity with dynamical torsion described in terms of a zweibein $e_{\mu}^{i}$ and a Lorentz connection $\omega_{\mu}$ by the action [50]

$$
S_{0}(e, \omega)=\int d^{2} x e\left(\frac{1}{16 \alpha} R_{\mu v}{ }^{i j} R^{\mu v}{ }_{i j}-\frac{1}{8 \beta} T_{\mu v}{ }^{i} T^{\mu v}{ }_{i}-\gamma\right),
$$

where $\alpha, \beta, \gamma$ are constant parameters, and the following notation is used:

$$
\begin{aligned}
e & =\operatorname{det} e_{\mu}^{i}, \\
R_{\mu v}{ }^{i j} & =\varepsilon^{i j} R_{\mu v}, \quad R_{\mu v}=\partial_{\mu} \omega_{v}-(\mu \leftrightarrow v), \\
T_{\mu v}{ }^{i} & =\partial_{\mu} e_{v}^{i}+\varepsilon^{i j} \omega_{\mu} e_{v j}-(\mu \leftrightarrow v) .
\end{aligned}
$$

Here, the indices of quantities transforming under the local Lorentz group are denoted by Latin characters, $i, j, k \ldots(i=0,1)$, with $\varepsilon^{i j}$ being a constant antisymmetric second-rank pseudo-tensor subject to the normalization $\varepsilon^{01}=1$. The indices of quantities transforming as (pseudo-)tensors under the general coordinate transformations are labelled by Greek characters, $\lambda, \mu, v \ldots(\lambda=0,1)$. The Latin 
indices are raised and lowered by the Minkowski metric $\eta_{i j}(+,-)$, and the Greek indices, by the metric tensor $g_{\mu v}=\eta_{i j} e_{\mu}^{i} e_{v}^{j}$. The action (46) is invariant under the local Lorentz transformations, $e_{\mu}^{i} \rightarrow e_{\mu}^{\prime}, \omega_{\mu} \rightarrow \omega^{\prime} \mu$

$$
\begin{aligned}
e_{\mu}^{\prime i} & =\left(\Lambda e_{\mu}\right)^{i}, \\
\left(\Omega_{\mu}^{\prime}\right)_{j}^{i} & =\left(\Lambda \Omega_{\mu} \Lambda^{-1}\right)_{j}^{i}+\left(\Lambda \partial_{\mu} \Lambda^{-1}\right)_{j}^{i}, \quad\left(\Omega_{\mu}\right)_{j}^{i} \equiv \varepsilon^{i k} \eta_{k j} \omega_{\mu},
\end{aligned}
$$

or, infinitesimally, with a parameter $\zeta$,

$$
\delta_{\zeta} e_{\mu}^{i}=\varepsilon^{i j} e_{\mu j} \zeta, \quad \delta_{\zeta} \omega_{\mu}=-\partial_{\mu} \zeta
$$

as well as under the general coordinate transformations, $x \rightarrow x^{\prime}=x^{\prime}(x)$,

$$
\begin{aligned}
e_{\mu}^{i} & \rightarrow e_{\mu}^{\prime i}\left(x^{\prime}\right)=\frac{\partial x^{\lambda}}{\partial x^{\prime} \mu} e_{\lambda}^{i}(x), \\
\omega_{\mu} & \rightarrow \omega_{\mu}^{\prime}\left(x^{\prime}\right)=\frac{\partial x^{\lambda}}{\partial x^{\prime} \mu} \omega_{\lambda}(x),
\end{aligned}
$$

implying the infinitesimal field variations, with some parameters $\xi^{\mu}$,

$$
\delta_{\xi} e_{\mu}^{i}=e_{\nu}^{i} \partial_{\mu} \xi^{v}+\left(\partial_{\nu} e_{\mu}^{i}\right) \xi^{v}, \quad \delta_{\xi} \omega_{\mu}=\omega_{\nu} \partial_{\mu} \xi^{v}+\left(\partial_{\nu} \omega_{\mu}\right) \xi^{v}
$$

The given model can be quantized using the Faddeev-Popov method, since the gauge transformations (49), (51) form a closed algebra:

$$
\begin{aligned}
{\left[\delta_{\zeta(1)}, \delta_{\zeta(2)}\right] } & =0, \\
{\left[\delta_{\xi(1)}, \delta_{\xi(2)}\right] } & =\delta_{\xi(1,2)}, \\
{\left[\delta_{\zeta}, \delta_{\xi}\right] } & =\delta_{\zeta^{\prime}},
\end{aligned}
$$

where

$$
\xi^{\mu}{ }_{(1,2)}=\xi^{v}{ }_{(1)} \partial_{\nu} \xi^{\mu}{ }_{(2)}-\left(\partial_{\nu} \xi^{\mu}{ }_{(1)}\right) \xi^{\nu}{ }_{(2)}, \quad \zeta^{\prime}=\left(\partial_{\mu} \zeta\right) \xi^{\mu} .
$$

In [75], a quantum theory for the gauge model (46), (49), (51), (52) has been presented according to the background field method, by using an ansatz for the vacuum functional which corresponds to $Z(B)$ of (43) for a Yang-Mills theory with the Nakanishi-Lautrup fields removes using a background gauge, see also [5]. Thus, the initial classical fields are ascribed the sets of quantum $Q$ and background $B$ fields, to be denoted by $Q=\left(q_{\mu}^{i}, q_{\mu}\right)$ and $B=\left(e_{\mu}^{i}, \omega_{\mu}\right)$, with the expressions for $g_{\mu \nu}$, as well as $e_{,}\left(\Omega_{\mu}\right)_{j}^{i}$ in (47), (48), being related only to the background fields. One also associates the gauge transformations (49), (51) with two forms of infinitesimal transformations, background $\delta_{\mathrm{b}}$ and quantum $\delta_{\mathrm{q}}$, being constructed by analogy with (44), so that the action $S_{0}(Q+B)$ in (46) remains invariant under both of these forms of transformations,

$$
\begin{aligned}
& \delta_{\mathrm{b}} e_{\mu}^{i}=\varepsilon^{i j} e_{\mu j} \zeta+e_{\nu}^{i} \partial_{\mu} \xi^{v}+\left(\partial_{\nu} e_{\mu}^{i}\right) \xi^{v}, \quad \delta_{\mathrm{b}} q_{\mu}^{i}=\varepsilon^{i j} q_{\mu j} \zeta+q_{\nu}^{i} \partial_{\mu} \xi^{v}+\left(\partial_{\nu} q_{\mu}^{a}\right) \xi^{v}, \\
& \delta_{\mathrm{b}} \omega_{\mu}=-\partial_{\mu} \zeta+\omega_{\nu} \partial_{\mu} \xi^{v}+\left(\partial_{\nu} \omega_{\mu}\right) \xi^{v}, \quad \delta_{\mathrm{b}} q_{\mu}=q_{\nu} \partial_{\mu} \xi^{v}+\left(\partial_{\nu} q_{\mu}\right) \xi^{v}, \\
& \delta_{\mathrm{q}} e_{\mu}^{i}=0, \quad \delta_{\mathrm{q}} q_{\mu}^{i}=\varepsilon^{i j}\left(e_{\mu j}+q_{\mu j}\right) \zeta+\left(e_{\nu}^{i}+q_{\nu}^{i}\right) \partial_{\mu} \xi^{v}+\left(\partial_{\nu} e_{\mu}^{i}+\partial_{\nu} q_{\mu}^{i}\right) \xi^{v}, \\
& \delta_{\mathrm{q}} \omega_{\mu}=0, \quad \delta_{\mathrm{q}} q_{\mu}=-\partial_{\mu} \zeta+\left(\omega_{\nu}+q_{\nu}\right) \partial_{\mu} \xi^{v}+\left(\partial_{\nu} \omega_{\mu}+\partial_{\nu} q_{\mu}\right) \xi^{v} .
\end{aligned}
$$

One next introduces the Faddeev-Popov ghosts $(\bar{c}, c),\left(\bar{c}^{\mu}, c^{\mu}\right)$ according to the respective number of gauge parameters $\zeta$, $\xi^{\mu}$ in (49), (51),

$$
\epsilon\left(\bar{c}, \bar{c}^{\mu}, c, c^{\mu}\right)=1, \operatorname{gh}\left(c, c^{\mu}\right)=-\operatorname{gh}\left(\bar{c}, \bar{c}^{\mu}\right)=1,
$$


and considers an analogue [5] of the generating functional of Green's functions, $\left(\bar{c}, \bar{c}^{\mu}, c, c^{\mu}\right)=(\bar{C}, C)$,

$$
Z(B, J)=\int d Q d \bar{C} d C \exp \left\{\frac{i}{\hbar}\left[S_{0}(Q+B)+S_{\mathrm{gf}}(Q, B)+S_{\mathrm{gh}}(Q, B ; \bar{C}, C)+J Q\right]\right\},
$$

where $J=\left(J_{i}^{\mu}, J^{\mu}\right)$ are sources to the quantum fields $Q=\left(q_{\mu}^{i}, q_{\mu}\right)$, and the functional $S_{\mathrm{gf}}=S_{\mathrm{gf}}(Q, B)$ is constructed using some background gauge functions $\chi, \chi_{\mu}$ (for the respective gauge parameters $\left.\zeta, \xi^{\mu}\right)$ as one demands its invariance, $\delta_{\mathrm{b}} S_{\mathrm{gf}}=0$, under the background transformations, whereas the ghost term $S_{\mathrm{gh}}=S_{\mathrm{gh}}(Q, B ; \bar{C}, C)$ is then determined by

$$
S_{\mathrm{gh}}=\left.\int d^{2} x\left(\bar{c} \delta_{\mathrm{q}} \chi+\bar{c}^{\mu} \delta_{\mathrm{q}} \chi_{\mu}\right)\right|_{\left(\zeta, \xi^{\mu}\right) \rightarrow\left(c, c^{\mu}\right)} .
$$

Let us choose the gauge functions $\chi=\chi(Q, B)$ and $\chi_{\mu}=\chi_{\mu}(Q, B)$ to be linear in the quantum fields $Q=\left(q_{\mu}^{i}, q_{\mu}\right)$ as in [75],

$$
\chi=e g^{\mu \nu} \nabla_{\mu} q_{v}, \quad \chi_{\mu}=e g^{\lambda v} e_{\mu i} \nabla_{\lambda} q_{v}^{i}, \quad g^{\mu \lambda} g_{\lambda v}=\delta_{v}^{\mu}
$$

where $e=\operatorname{det} e_{\mu}^{i}, g_{\mu \nu}=\eta_{i j} e_{\mu}^{i} e_{v}^{j}$, and $\nabla_{\mu}$ is a covariant derivative acting on an arbitrary (psedo-)tensor field $T_{\mu_{1} \ldots \mu_{k} i_{1} \ldots i_{m}}^{v_{1} \ldots \nu_{l} j_{1} \ldots j_{n}}$, in terms of the connection $\left(\Omega_{\mu}\right)_{j}^{i}=\varepsilon^{i k} \eta_{k j} \omega_{\mu}$ and the Christoffel symbols $\Gamma_{\mu v}^{\lambda}$

$$
\Gamma_{\mu v}^{\lambda}=\frac{1}{2} g^{\lambda \sigma}\left(\partial_{\nu} g_{\mu \sigma}+\partial_{\mu} g_{v \sigma}-\partial_{\sigma} g_{\mu v}\right)
$$

by the rule

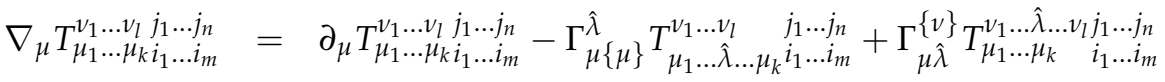

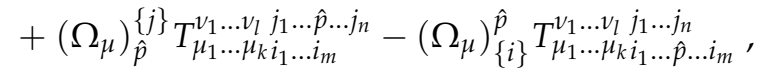

with the shorthand notation, $p_{k} \in\left\{p_{1}, \ldots, p_{m}\right\}, v_{k} \in\left\{v_{1}, \ldots, v_{n}\right\}$,

$$
\begin{aligned}
F_{\{i\}}^{\hat{p}} T_{i_{1} \cdots \hat{p} \cdots i_{m}}^{\mu_{1} \cdots \mu_{n}} & =\sum_{p_{k}} F_{i_{k}}^{p_{k}} T_{i_{1} \cdots p_{k} \cdots i_{m}}^{\mu_{1} \cdots \mu_{n}}, \quad G_{\hat{v}}^{\{\mu\}} T_{i_{1} \cdots i_{m}}^{\mu_{1} \cdots \hat{v} \cdots \mu_{n}}=\sum_{v_{k}} G_{v_{k}}^{\mu_{k}} T_{i_{1} \cdots i_{m}}^{\mu_{1} \cdots v_{k} \cdots \mu_{n}}, \\
F_{\hat{p}}^{\{i\}} T_{\mu_{1} \cdots \mu_{n}}^{i_{1} \cdots \hat{p} \cdots i_{m}} & =\sum_{p_{k}} F_{p_{k}}^{i_{k}} T_{\mu_{1} \cdots \mu_{n}}^{i_{1} \cdots p_{k} \cdots i_{m}}, \quad G_{\{\mu\}}^{\hat{v}} T_{\mu_{1} \cdots \hat{v} \cdots \mu_{n}}^{i_{1} \cdots i_{m}}=\sum_{v_{k}} G_{\mu_{k}}^{v_{k}} T_{\mu_{1} \cdots v_{k} \cdots \mu_{n}}^{i_{1} \cdots i_{m}},
\end{aligned}
$$

so that the covariant derivative $\nabla_{\mu}$ features the standard properties (with $F$, $G$ being arbitrary (psedo-)tensor fields)

$$
\nabla_{\sigma} g_{\mu \nu}=\nabla_{\sigma} g^{\mu \nu}=0, \quad \nabla_{\mu}(F G)=F \nabla_{\mu} G+\left(\nabla_{\mu} F\right) G .
$$

The above objects make it possible to construct the gauge-fixing term $S_{\mathrm{gf}}$ as a functional quadratic in the background gauges $\chi, \chi^{\mu}$ (including some numeric parameters $a, b$ )

$$
S_{\mathrm{gf}}=\frac{1}{2} \int d^{2} x e^{-1}\left(a \chi^{2}+b \chi_{\mu} \chi^{\mu}\right)
$$

and invariant under the local Lorentz transformations

$$
\begin{aligned}
e_{\mu}^{\prime i} & =\left(\Lambda e_{\mu}\right)^{i}, q_{\mu}^{\prime i}=\left(\Lambda q_{\mu}\right)^{i}, \\
\left(\Omega_{\mu}^{\prime}\right)_{b}^{i} & =\left(\Lambda \Omega_{\mu} \Lambda^{-1}\right)_{j}^{i}+\left(\Lambda \partial_{\mu} \Lambda^{-1}\right)_{j}^{i}, \quad q_{\mu}^{\prime}=q_{\mu},
\end{aligned}
$$


as well as under the general coordinate transformations, $x \rightarrow x^{\prime}=x^{\prime}(x)$,

$$
\begin{array}{ll}
e_{\mu}^{\prime}\left(x^{\prime}\right)=\frac{\partial x^{\lambda}}{\partial x^{\prime} \mu} e_{\lambda}^{i}(x), \quad \omega_{\mu}^{\prime}\left(x^{\prime}\right)=\frac{\partial x^{\lambda}}{\partial x^{\prime} \mu} \omega_{\lambda}(x), \\
q_{\mu}^{\prime i}\left(x^{\prime}\right)=\frac{\partial x^{\lambda}}{\partial x^{\prime} \mu} q_{\lambda}^{i}(x), \quad q_{\mu}^{\prime}\left(x^{\prime}\right)=\frac{\partial x^{\lambda}}{\partial x^{\prime} \mu} q_{\lambda}(x) .
\end{array}
$$

The field transformations (63) and (64) coincide infinitesimally with the background transformations (53), which thereby implies the fulfillment of $\delta_{\mathrm{b}} S_{\mathrm{gf}}=0$. Having this in mind, as well as the fact that the non-zero quantum transformations (54), with account taken of (59), can be represented as

$$
\begin{aligned}
\delta_{\mathrm{q}} q_{\mu}^{i} & =\varepsilon^{i j}\left(e_{\mu j}+q_{\mu j}\right) c+\left(e_{\nu}^{i}+q_{v}^{i}\right) \nabla_{\mu} c^{v}+\left(\nabla_{\nu} e_{\mu}^{i}+\nabla_{\nu} q_{\mu}^{i}\right) c^{v}-\varepsilon^{i j} \omega_{\nu}\left(e_{\mu j}+q_{\mu b}\right) c^{v}, \\
\delta_{\mathrm{q}} q_{\mu} & =-\nabla_{\mu} c+\left(\omega_{\nu}+q_{v}\right) \nabla_{\mu} c^{v}+\left(\nabla_{\nu} \omega_{\mu}+\nabla_{\nu} q_{\mu}\right) c^{v}
\end{aligned}
$$

under $\zeta \rightarrow c, \xi^{\mu} \rightarrow c^{\mu}$, the ghost contribution $S_{\mathrm{gh}}$ in (56) reads

$$
\begin{aligned}
S_{\mathrm{gh}}= & \int d^{2} x e\left\{-\bar{c} \nabla_{\mu} \nabla^{\mu} c+\bar{c} \nabla^{\mu}\left[\left(\nabla_{\nu} \omega_{\mu}+\nabla_{\nu} q_{\mu}\right) c^{\nu}+\left(\omega_{\nu}+q_{\nu}\right) \nabla_{\mu} c^{\nu}\right]\right. \\
& +\varepsilon^{i j} \bar{c}^{\mu} e_{\mu i} \nabla^{\nu}\left[\left(e_{v j}+q_{v j}\right)\left(c-\omega_{\lambda} c^{\lambda}\right)\right] \\
& \left.+\bar{c}^{\mu} e_{\mu i} \nabla^{v}\left[\left(\nabla_{\lambda} e_{v}^{i}+\nabla_{\lambda} q_{\nu}^{i}\right) c^{\lambda}+\left(e_{\lambda}^{i}+q_{\lambda}^{i}\right) \nabla_{\nu} c^{\lambda}\right]\right\} .
\end{aligned}
$$

The quantum action in (55) represented by (46), (57), (62), (65) turns out to be invariant (which is also valid for the integrand in (55) once the sources are put to zero $J=0$ under the assumption $\delta(x)=\left.\partial_{\mu} \delta(x)\right|_{x=0}=0$ ) with respect to the background transformations (53) accompanied by a set of compensating local transformations for the ghost fields [75],

$$
\begin{array}{ll}
\delta \bar{c}=\left(\partial_{\mu} \bar{c}\right) \xi^{\mu}, & \delta \bar{c}^{\mu}=-\bar{c}^{v} \partial_{\nu} \xi^{\mu}+\left(\partial_{\nu} \bar{c}^{\mu}\right) \xi^{v}, \\
\delta c=-c^{\mu} \partial_{\mu} \zeta+\left(\partial_{\mu} c\right) \xi^{\mu}, & \delta c^{\mu}=-c^{v} \partial_{\nu} \xi^{\mu}+\left(\partial_{\nu} c^{\mu}\right) \xi^{v} .
\end{array}
$$

From (53) and (66), it follows that the generating functional $Z(B, J)$ in (55) is invariant [75] under the initial gauge transformations (49), (51) of the background fields $B=\left(e_{\mu}^{i}, \omega_{\mu}\right)$ along with the following local transformations of the sources $J=\left(J_{i}^{\mu}, J^{\mu}\right)$ :

$$
\delta J_{i}^{\mu}=-\varepsilon_{i}^{k} J_{k}^{\mu} \zeta-J_{i}^{v} \partial_{\nu} \xi^{\mu}+\partial_{v}\left(J_{i}^{\mu} \xi^{v}\right), \quad \delta J^{\mu}=-J^{v} \partial_{\nu} \xi^{\mu}+\partial_{v}\left(J^{\mu} \xi^{v}\right), \quad \varepsilon_{j}^{i} \equiv \varepsilon^{i k} \eta_{k j},
$$

which implies the property

$$
\delta(J Q)=\int d^{2} x \partial_{\mu} F^{\mu}, \quad F^{\mu}(x) \equiv\left(J_{i}^{v} q_{v}^{i}+J^{v} q_{v}\right) \xi^{\mu}
$$

for the source term $J Q$ in (55) and extends an infinitesimal tensor transformation law for the sources $J_{i}^{\mu}$ and $J^{\mu}$ by adding the contributions $J_{i}^{\mu} \partial_{\nu} \xi^{\nu}$ and $J^{\mu} \partial_{\nu} \xi^{\nu}$, indeed,

$$
\delta J_{i}^{\mu}=\left[-\varepsilon_{i}^{k} J_{k}^{\mu} \zeta-J_{i}^{v} \partial_{\nu} \xi^{\mu}+\left(\partial_{\nu} J_{i}^{\mu}\right) \xi^{\nu}\right]+J_{i}^{\mu} \partial_{\nu} \xi^{\nu}, \quad \delta J^{\mu}=\left[-J^{v} \partial_{\nu} \xi^{\mu}+\left(\partial_{\nu} J^{\mu}\right) \xi^{v}\right]+J^{\mu} \partial_{\nu} \xi^{\nu} .
$$

Given the invariance of $Z(B, J)=\exp \{(i / h) W(B, J)\}$ under (49), (51), (67), one establishes the invariance [75] of a functional $\Gamma=\Gamma(B, Q)$ defined as

$$
\Gamma(B, Q)=W(B, J)-J Q, \quad Q=\frac{\delta W}{\delta J}, \quad J=-\frac{\delta \Gamma}{\delta Q}, \quad Q=\left(q_{\mu}^{i}, q_{\mu}\right)
$$


under the background transformations (53) of the fields $B$ and $Q$,

$$
\delta_{\mathrm{b}} \Gamma=\int d^{2} x\left[\frac{\delta \Gamma}{\delta B(x)} \delta_{\mathrm{b}} B(x)+\frac{\delta \Gamma}{\delta Q(x)} \delta_{\mathrm{b}} Q(x)\right]=0,
$$

which entails that the effective action $\Gamma_{\text {eff }}(B)$ of the background field method,

$$
\Gamma_{\text {eff }}(B)=\left.\Gamma(B, Q)\right|_{Q=0},
$$

proves to be invariant, $\delta_{(\zeta, \xi)} \Gamma_{\text {eff }}=0$, under the gauge transformations (49), (51) of the background fields $B=\left(e_{\mu}^{i}, \omega_{\mu}\right)$.

\section{Composite Field Introduction}

Let us extend the generating functional (55) proposed in [75] to a functional $Z(B, J, L)$, as we introduce a set of local background-dependent composite fields $\sigma^{m}(Q, B)$ with sources $L_{m}$,

$$
\sigma^{m}(Q, B)=\sigma_{\mu_{1} \cdots \mu_{l}}^{i_{1} \cdots i_{k}}(Q(x), B(x)), L_{m}=L_{i_{1} \cdots i_{k}}^{\mu_{1} \cdots \mu_{l}}(x), \quad m=\left(x, i_{1}, \ldots, i_{k}, \mu_{1}, \ldots, \mu_{l}\right),
$$

and denote the entire quantum action by $S(Q, B ; \bar{C}, C)$,

$$
Z(B, J, L)=\int d Q d \bar{C} d C \exp \left\{\frac{i}{\hbar}\left[S(Q, B ; \bar{C}, C)+J Q+L_{m} \sigma^{m}(Q, B)\right]\right\} .
$$

In this setting, we demand that the extended functional $Z(B, J, L)$ inherit the local symmetry of $Z(B, J)$ under the transformations (49), (51), (67) of the background fields $B=\left(e_{\mu}^{i}, \omega_{\mu}\right)$ and the sources $J=\left(J_{i}^{\mu}, J^{\mu}\right)$. To do so, we impose the condition that the composite fields $\sigma_{\mu_{1} \cdots \mu_{n}}^{i_{1} \cdots i_{m}}(x)=$ $\sigma_{\mu_{1} \cdots \mu_{n}}^{i_{1} \cdots i_{m}}(Q(x), B(x))$ should behave as tensors with respect to the Lorentz (63) and general coordinate (64) transformations of the quantum and background fields,

$$
\begin{aligned}
& \sigma_{\mu_{1} \cdots \mu_{n}}^{i_{1} \cdots i_{m}}(x)=\Lambda_{j_{1}}^{i_{1}} \cdots \Lambda_{j_{m}}^{i_{m}} \sigma_{\mu_{1} \cdots \mu_{n}}^{j_{1} \cdots j_{m}}(x), \\
& \sigma_{\mu_{1} \cdots \mu_{n}}^{\prime i_{1} \cdots i_{m}}\left(x^{\prime}\right)=\frac{\partial x^{\nu_{1}}}{\partial x^{\prime} \mu_{1}} \cdots \frac{\partial x^{\nu_{n}}}{\partial x^{\prime} \mu_{n}} \sigma_{v_{1} \cdots v_{n}}^{i_{1} \cdots i_{m}}(x), \quad x^{\prime}=x^{\prime}(x) \text {. }
\end{aligned}
$$

Generally, a composite field $\sigma_{\mu_{1} \cdots \mu_{n}}^{i_{1} \cdots i_{m}}(Q, B)$ transforming as (70) is multiplicative with respect to the quantum fields $Q=\left(q_{\mu}^{i}, q_{\mu}\right)$ and the background field objects $e_{\mu}^{i}, g_{\mu v}, R_{\mu \nu}{ }^{i j}, T_{\mu \nu}{ }^{i}$, see (47). Such a field may also include some background covariant derivatives $\nabla_{\mu}$ acting as (59), (58) and having the properties (61). Given this, any composite field limited by $\sigma_{\mu_{1} \cdots \mu_{n}}^{i_{1} \ldots i_{m}}(Q, 0) \neq 0$ is allowed to contain the background fields $B$ only via the covariant derivative $\nabla_{\mu}$, defined in terms of $\Gamma_{\mu v}^{\lambda},\left(\Omega_{\mu}\right)_{j}^{i}$ and acting on $q_{\mu}^{i}, q_{\mu}$ as follows:

$$
\nabla_{\mu} q_{\nu}^{i}=\partial_{\mu} q_{\nu}^{i}-\Gamma_{\mu \nu}^{\lambda} q_{\lambda}^{i}+\left(\Omega_{\mu}\right)_{j}^{i} q_{\nu}^{j}, \quad \nabla_{\mu} q_{\nu}=\partial_{\mu} q_{\nu}-\Gamma_{\mu \nu}^{\lambda} q_{\lambda}
$$

The transformations (70) correspond infinitesimally to local tensor variations $\delta \sigma_{\mu_{1} \cdots \mu_{n}}^{i_{1} \cdots i_{m}}$ with parameters $\zeta$ and $\xi^{\mu}$,

$$
\delta \sigma_{\mu_{1} \cdots \mu_{n}}^{i_{1} \cdots i_{m}}=\varepsilon_{\hat{p}}^{\{i\}} \sigma_{\mu_{1} \cdots \mu_{n}}^{i_{1} \cdots \hat{p} \cdots i_{m}} \zeta+\sigma_{\mu_{1} \cdots \hat{v} \cdots \mu_{n}}^{i_{1} \cdots i_{m}} \partial_{\{\mu\}} \xi^{\hat{v}}+\left(\partial_{\nu} \sigma_{\mu_{1} \cdots \mu_{n}}^{i_{1} \cdots i_{m}}\right) \xi^{v} .
$$

Under these assumptions and the invariance of the vacuum functional in (69) with respect to the background transformations (53), accompanied by the compensating local transformations (66) of the ghost fields, the modified generating functional $Z(B, J, L)$ in (69) is invariant under the initial gauge 
transformations (49), (51) of the background fields $B=\left(e_{\mu}^{i}, \omega_{\mu}\right)$ along with the local transformations (67) of the sources $J=\left(J_{i}^{\mu}, J^{\mu}\right)$ and some local transformations of the sources $L_{i_{1} \cdots i_{m}}^{\mu_{1} \cdots \mu_{n}}$,

$$
\delta L_{i_{1} \cdots i_{m}}^{\mu_{1} \cdots \mu_{n}}=-\varepsilon_{\{i\}}^{\hat{p}} L_{i_{1} \cdots \hat{p} \cdots i_{m}}^{\mu_{1} \cdots \mu_{n}} \zeta-L_{i_{1} \cdots i_{m}}^{\mu_{1} \cdots \hat{v} \cdots \mu_{n}} \partial_{\hat{v}} \xi^{\{\mu\}}+\partial_{\nu}\left(L_{i_{1} \cdots i_{m}}^{\mu_{1} \cdots \mu_{n}} \xi^{v}\right),
$$

which diverges from the infinitesimal tensor transformation law by the contribution $L_{i_{1} \cdots i_{m}}^{\mu_{1} \cdots \mu_{n}} \partial_{\nu} \xi^{v}$,

$$
\delta L_{i_{1} \cdots i_{m}}^{\mu_{1} \cdots \mu_{n}}=\left[-\varepsilon_{\{i\}}^{\hat{p}} L_{i_{1} \cdots \hat{p} \cdots i_{m}}^{\mu_{1} \cdots \mu_{n}} \zeta-L_{i_{1} \cdots i_{m}}^{\mu_{1} \cdots \hat{v} \cdots \mu_{n}} \partial_{\hat{\nu}} \xi^{\{\mu\}}+\left(\partial_{\nu} L_{i_{1} \cdots i_{m}}^{\mu_{1} \cdots \mu_{n}}\right) \zeta^{\nu}\right]+L_{i_{1} \cdots i_{m}}^{\mu_{1} \cdots \mu_{n}} \partial_{\nu} \zeta^{\nu}
$$

and provides for the source term $L_{m} \sigma^{m}$ in (69) the transformation property

$$
\delta\left(L_{m} \sigma^{m}\right)=\int d^{2} x \partial_{\mu} G^{\mu}, \quad G^{\mu} \equiv L_{i_{1} \cdots i_{m}}^{v_{1} \cdots v_{n}} \sigma_{v_{1} \cdots v_{n}}^{i_{1} \cdots i_{m}} \xi^{\mu} .
$$

The invariance of $Z(B, J, L)$ under (49), (51), (67), (72) can be represented as

$$
\begin{aligned}
& \int d^{2} x\left\{\left[\varepsilon^{i j} e_{\mu j} \zeta+e_{\nu}^{i}\left(\partial_{\mu} \xi^{v}\right)+\left(\partial_{\nu} e_{\mu}^{i}\right) \xi^{v}\right] \frac{\delta}{\delta e_{\mu}^{i}}+\left[-\partial_{\mu} \zeta+\omega_{v}\left(\partial_{\mu} \xi^{v}\right)+\left(\partial_{\nu} \omega_{\mu}\right) \xi^{v}\right] \frac{\delta}{\delta \omega_{\mu}}\right. \\
& -\left[\varepsilon_{i}^{k} J_{k}^{\mu} \zeta+J_{i}^{v}\left(\partial_{\nu} \xi^{\mu}\right)-\partial_{\nu}\left(J_{i}^{\mu} \xi^{v}\right)\right] \frac{\delta}{\delta J_{i}^{\mu}}-\left[J^{\nu}\left(\partial_{\nu} \xi^{\mu}\right)-\partial_{\nu}\left(J^{\mu} \xi^{v}\right)\right] \frac{\delta}{\delta J^{\mu}} \\
& \left.-\left[\varepsilon_{\{i\}}^{\hat{p}} L_{i_{1} \cdots \hat{p} \cdots i_{m}}^{\mu_{1} \cdots \mu_{n}} \zeta+L_{i_{1} \cdots i_{m}}^{\mu_{1} \cdots \hat{\nu_{n}} \cdots}\left(\partial_{\hat{\nu}} \xi^{\{\mu\}}\right)-\partial_{\nu}\left(L_{i_{1} \cdots i_{m}}^{\mu_{1} \cdots \mu^{n}} \xi^{v}\right)\right] \frac{\delta}{\delta L_{i_{1} \cdots i_{m}}^{\mu_{1} \cdots \mu_{n}}}\right\} Z(B, J, L)=0 .
\end{aligned}
$$

Let us introduce a functional $\Gamma=\Gamma(B, Q, \Sigma)$ given by the double Legendre transformation

$$
\Gamma(B, Q, \Sigma)=W(B, J, L)-J Q-L_{m}\left[\sigma^{m}(Q, B)+\Sigma^{m}\right], W=(\hbar / i) \ln Z
$$

in terms of additional fields $\Sigma^{m}=\sum_{\mu_{1} \cdots \mu_{n}}^{i_{1} \cdots i_{m}}(x)$,

$$
Q=\frac{\delta W}{\delta J}, \Sigma^{m}=\frac{\delta W}{\delta L_{m}}-\sigma^{m}\left(\frac{\delta W}{\delta J}, B\right),-J=\frac{\delta \Gamma}{\delta Q}+L_{m} \frac{\delta \sigma^{m}}{\delta Q},-L_{m}=\frac{\delta \Gamma}{\delta \Sigma^{m}} .
$$

The effective action $\Gamma_{\text {eff }}(B, \Sigma)$ with composite and background fields,

$$
\Gamma_{\text {eff }}(B, \Sigma)=\left.\Gamma(B, Q, \Sigma)\right|_{Q=0},
$$

then satisfies the identity

$$
\begin{aligned}
& \int d^{2} x\left\{\left[\varepsilon^{i j} e_{\mu j} \zeta+e_{\nu}^{i}\left(\partial_{\mu} \xi^{v}\right)+\left(\partial_{\nu} e_{\mu}^{i}\right) \xi^{\nu}\right] \frac{\delta}{\delta e_{\mu}^{i}}+\left[-\partial_{\mu} \zeta+\omega_{\nu}\left(\partial_{\mu} \xi^{\nu}\right)+\left(\partial_{\nu} \omega_{\mu}\right) \xi^{v}\right] \frac{\delta}{\delta \omega_{\mu}}\right. \\
& \left.+\left[\varepsilon_{\{i\}}^{\hat{p}} \sum_{\mu_{1} \cdots \mu_{n}}^{i_{1} \cdots i_{m}} \zeta \frac{\delta}{\delta \Sigma_{\mu_{1} \cdots \mu_{n}}^{i_{1} \cdots i_{m}}}+\sum_{\mu_{1} \cdots \mu_{n}}^{i_{1} \cdots i_{m}}\left(\partial_{\nu} \xi^{\{\mu\}}\right) \frac{\delta}{\delta \Sigma_{\mu_{1} \cdots \cdots \mu_{n}}^{i_{1} \cdots \cdots}}+\left(\partial_{\nu} \sum_{\mu_{1} \cdots \mu_{n}}^{i_{1} \cdots i_{m}}\right) \xi^{v} \frac{\delta}{\delta \Sigma_{\mu_{1} \cdots \cdots n_{n}}^{i_{1} \cdots m_{m}}}\right]\right\} \Gamma_{\text {eff }}(B, \Sigma)=0,
\end{aligned}
$$

due to integration by parts in (73), as written in terms of $\Gamma(B, Q, \Sigma)$, which is completed by setting $Q=\sigma^{m}(0, B)=0$. Using the latter identity for $\Gamma_{\text {eff }}(B, \Sigma)$ and the following consequences, cf. (35), of the notation (60),

$$
\begin{gathered}
\varepsilon_{\{i\}}^{\hat{p}} \sum_{\mu_{1} \cdots \mu_{n}}^{i_{1} \cdots i_{m}} \frac{\delta}{\delta \sum_{\mu_{1} \cdots \mu_{n}}^{i_{1} \cdots \hat{p} \cdots i_{m}}}=\varepsilon_{\hat{p}}^{\{i\}} \sum_{\mu_{1} \cdots \mu_{n}}^{i_{1} \cdots \hat{p} \cdots i_{m}} \frac{\delta}{\delta \sum_{\mu_{1} \cdots \mu_{n}}^{i_{1} \cdots i_{m}}}, \\
\sum_{\mu_{1} \cdots \mu_{n}}^{i_{1} \cdots i_{m}}\left(\partial_{\hat{\nu}} \xi^{\{\mu\}}\right) \frac{\delta}{\delta \sum_{\mu_{1} \cdots i_{m} \cdots \mu_{n}}^{i_{1} \cdots i_{n}}}=\sum_{\mu_{1} \cdots \hat{v} \cdots \mu_{n}}^{i_{1} \cdots i_{m}}\left(\partial_{\{\mu\}} \xi^{\hat{v}}\right) \frac{\delta}{\delta \Sigma_{\mu_{1} \cdots \mu_{n}}^{i_{1} \cdots i_{m}}},
\end{gathered}
$$


by virtue of $\varepsilon_{k}^{j}=\varepsilon^{j i} \eta_{i k}=\varepsilon_{j}^{k}\left(\varepsilon_{1}^{0}=\varepsilon_{0}^{1}=-1, \varepsilon_{0}^{0}=\varepsilon_{1}^{1}=0\right)$, we find that $\Gamma_{\text {eff }}(B, \Sigma)$ is invariant, $\delta_{(\zeta, \xi)} \Gamma_{\text {eff }}=0$, under a set of local transformations comprised by the gauge transformations (49), (51) of the background fields $B=\left(e_{\mu}^{i}, \omega_{\mu}\right)$ along with the infinitesimal local tensor transformations

$$
\delta_{(\zeta, \xi)} \sum_{\mu_{1} \cdots \mu_{n}}^{i_{1} \cdots i_{m}}=\varepsilon_{\hat{p}}^{\{i\}} \sum_{\mu_{1} \cdots \mu_{n}}^{i_{1} \cdots \hat{p} \cdots i_{m}} \zeta+\Sigma_{\mu_{1} \cdots \hat{v} \cdots \mu_{n}}^{i_{1} \cdots i_{m}} \partial_{\{\mu\}} \xi^{\hat{v}}+\left(\partial_{v} \sum_{\mu_{1} \cdots \mu_{n}}^{i_{1} \cdots i_{m}}\right) \xi^{v}
$$

of the additional fields $\Sigma_{\mu_{1} \cdots \mu_{n}}^{i_{1} \cdots i_{m}}$, cf. (70), (71).

\section{Gribov-Zwanziger Model}

Let us examine the Gribov-Zwanziger model $[23,24]$ implementing the concept of Gribov horizon [22] in quantum Yang-Mills theories by using a non-local composite field. To this end, we consider a Euclidean form of the Faddeev-Popov action $S_{\mathrm{FP}}(\phi)$ in Landau gauge $\chi_{\mathrm{L}}^{p}(\phi)=\partial^{\mu} A_{\mu}^{p}$, (8), (9), (10), and examine a non-local horizon functional $H(A)$,

$$
H(A)=\gamma \int d^{D} x\left[\int d^{D} y f^{p r t} g A_{\mu}^{r}(x)\left(K^{-1}\right)^{p q}(x ; y) f^{q s t} g A^{s \mid \mu}(y)+D\left(N^{2}-1\right)\right],
$$

where, in Euclidean metric $A_{\mu}=A^{\mu}$, we use the signature $\eta_{\mu v}=(-,+, \ldots,+)$ under the Wick rotation $x^{0} \rightarrow i x^{0}, A^{p \mid 0} \rightarrow i A^{p \mid 0}, S_{\mathrm{FP}} \rightarrow i S_{\mathrm{FP}}$ and maintain the summation convention $A_{\mu} B_{\mu}=A_{\mu} B^{\mu}$; besides, $\mathrm{K}^{-1}$ is the inverse,

$$
\int d^{D} z\left(K^{-1}\right)^{p r}(x ; z)(K)^{r q}(z ; y)=\int d^{D} z(K)^{p r}(x ; z)\left(K^{-1}\right)^{r q}(z ; y)=\delta^{p q} \delta(x-y),
$$

of the Faddeev-Popov operator $K$ in terms of the gauge condition $\partial^{\mu} A_{\mu}^{p}=0$,

$$
K^{p q}(x ; y)=\left(\delta^{p q} \partial^{2}+g f^{p r q} A_{\mu}^{r} \partial^{\mu}\right) \delta(x-y), \quad K^{p q}(x ; y)=K^{q p}(y ; x),
$$

while $\gamma$ is a Gribov thermodynamic parameter [23,24], introduced in a self-consistent way by using a gap equation (horizon condition) for a Gribov-Zwanziger action $S_{\mathrm{GZ}}=S_{\mathrm{GZ}}(\phi)$,

$$
\frac{\partial E_{\mathrm{vac}}}{\partial \gamma}=0, \quad \exp \left(-\hbar^{-1} E_{\mathrm{vac}}\right) \equiv \int d \phi \exp \left(-\hbar^{-1} S_{\mathrm{GZ}}\right)
$$

where $E_{\mathrm{vac}}$ is the vacuum energy, and the action $S_{\mathrm{GZ}}$ reads

$$
S_{\mathrm{GZ}}(\phi)=S_{\mathrm{FP}}(\phi)-H(A) .
$$

A generating functional of Green's functions $Z_{H}(J)$ for the quantum theory under study can be given in terms of a Faddeev-Popov action shifted by a constant value, $S_{\mathrm{FP}}(\phi)-H(0)$,

$$
Z_{H}(J)=\left.Z_{H}(J, L)\right|_{L=1}, Z_{H}(J, L)=\int d \phi \exp \left\{-\hbar^{-1}\left[S_{\mathrm{FP}}(\phi)-H(0)+J_{A} \phi^{A}+L \sigma(A)\right]\right\},
$$

where $L=L(x), \epsilon(L)=\operatorname{gh}(L)=0$, is a source to the non-local composite field

$$
\sigma(A)(x)=\gamma \int d^{D} y f^{t r p} g A_{\mu}^{r}(x)\left(\tilde{K}^{-1}\right)^{p q}(x ; y) f^{q s t} g A^{s \mid \mu}(y),
$$

with $\tilde{K}^{-1}$ being the inverse,

$$
\int d^{D} z\left(\tilde{K}^{-1}\right)^{p r}(x ; z)(\tilde{K})^{r q}(z ; y)=\int d^{D} z(\tilde{K})^{p r}(x ; z)\left(\tilde{K}^{-1}\right)^{r q}(z ; y)=\delta^{p q} \delta(x-y),
$$


of an operator $\tilde{K}$ defined for a quantity $F^{p}=F^{p}(x)$,

$$
\int d^{D} y(\tilde{K})^{p q}(x ; y) F^{q}(y) \equiv\left[\partial_{\mu},\left[D^{\mu}(A), F\right]\right]^{p}(x), A_{\mu}(x)=T^{p} A_{\mu}^{p}(x), \quad F(x)=T^{p} F^{p}(x),
$$

which implies

$$
\tilde{K}^{p q}(x ; y)=\partial^{\mu} D_{\mu}^{p q}(A) \delta(x-y),
$$

and reduces to the operator $K$ of (79) under the Landau gauge condition due to $S_{\mathrm{FP}}(\phi)$ in the path integral (81).

\subsection{Formal Background Introduction}

Let us now formally extend the generating functional $Z_{H}(J, L)$ with a non-local composite field (81)-(84) to the case of an additional background field $B_{\mu}$ with a covariant derivative $D_{\mu}(B)$ and the gauge properties (16), (17), by using the approach (2), (19), as adapted to Euclidean QFT, which implies a modification of derivatives $\partial_{\mu} \rightarrow D_{\mu}(B)$ in (81), according to

$$
Z_{H}(B, J, L)=\left.Z_{H}(J, L)\right|_{\partial_{\mu} \rightarrow D_{\mu}(B)}=\int d \phi \exp \left\{-\hbar^{-1}\left[S_{\mathrm{FP}}(\phi, B)-H(0)+J_{A} \phi^{A}+L \sigma(A, B)\right]\right\},
$$

where $S_{\mathrm{FP}}(\phi, B)$ is the Faddeev-Popov action in the Landau background gauge $\chi_{\mathrm{L}}^{p}(\phi, B)=0$, see (41), and $\sigma(A, B)$ is a non-local composite field on a background,

$$
\sigma(A, B)(x)=\gamma \int d^{D} y f^{t r p} g A_{\mu}^{r}(x)\left(\tilde{K}_{B}^{-1}\right)^{p q}(x ; y) f^{q s t} g A^{s \mid \mu}(y) .
$$

Here, $\tilde{K}_{B}^{-1}$ is a modified operator $\tilde{K}^{-1}$ as in (78), with the related inverse $\tilde{K}_{B}$ given by the replacement $\tilde{K} \rightarrow \tilde{K}_{B}$,

$$
\int d^{D} y(\tilde{K})_{B}^{p q}(x ; y) F^{q}(y) \equiv\left[D_{\mu}(B),\left[D^{\mu}(A+B), F\right]\right]^{p}(x), \quad F(x)=F^{p}(x) T^{p},
$$

and having the explicit form

$$
(\tilde{K})_{B}^{p q}(x ; y)=D_{\mu}^{p r}(B) D^{r q \mid \mu}(A+B) \delta(x-y) .
$$

In the particular case, cf. (81),

$$
Z_{H}(B, J)=\left.Z_{H}(B, J, L)\right|_{L=1},
$$

we obtain the generating functional

$$
Z_{H}(B, J)=\int d \phi \exp \left\{-\hbar^{-1}\left[S_{\mathrm{GZ}}(\phi, B)+J_{A} \phi^{A}\right]\right\}, \quad S_{\mathrm{GZ}}(\phi, B) \equiv S_{\mathrm{FP}}(\phi, B)-H(A, B),
$$

with a non-local term $H(A, B)$ given by

$$
H(A, B)=\gamma \int d^{D} x\left[\int d^{D} y f^{p r t} g A_{\mu}^{r}(x)\left(K_{B}^{-1}\right)^{p q}(x ; y) f^{q s t} g A^{s \mid \mu}(y)+D\left(N^{2}-1\right)\right] .
$$

Here, $K_{B}^{-1}$ is the inverse of an operator $K_{B}$ as in (83), which is identical with $\tilde{K}_{B}$ in (88) being expressed, due to $S_{\mathrm{FP}}(\phi, B)$ in $(90)$, by utilizing the gauge condition $D_{\mu}^{p q}(B) A^{q \mid \mu}=0$ and the properties of $f^{p q r}$ including the Jacobi identity,

$$
K_{B}(x ; y)=\left[\partial^{2}+g\left(\partial_{\mu} B^{\mu}\right)+g\left(A_{\mu}+2 B_{\mu}\right) \partial^{\mu}+g^{2}\left(A_{\mu}+B_{\mu}\right) B^{\mu}\right] \delta(x-y),
$$


where $A_{\mu}, B_{\mu}$ are matrices with the elements $\left(A_{\mu}^{p q}, B_{\mu}^{p q}\right)=f^{p r q}\left(A_{\mu}^{r}, B_{\mu}^{r}\right)$, and $K_{B}(x ; y)$ is related to $\tilde{K}_{B}(x ; y)$ in (89) as follows:

$$
K_{B}(x ; y)=\tilde{K}_{B}(x ; y)-g\left[D_{\mu}(B), A^{\mu}\right] \delta(x-y)=D^{\mu}(A+B) D_{\mu}(B) \delta(x-y) .
$$

The operator $K_{B}$ extends the original operator $K$ in (79) and exhibits the properties

$$
\left.K_{B}\right|_{B=0}=K, \quad\left(K_{B}\right)^{p q}(x ; y)=\left(K_{B}\right)^{q p}(y ; x),
$$

which can be verified by a direct calculation:

$$
\int d^{D} y\left[\left(K_{B}\right)^{p q}(x ; y)-\left(K_{B}\right)^{q p}(y ; x)\right] F^{q}(y)=g f^{p r q}\left[D_{\mu}^{r s}(B) A^{s \mid \mu}\right] F^{q}(x)=0 .
$$

By virtue of (94), one may formally interpret $S_{\mathrm{GZ}}(\phi, B)$ as a Gribov-Zwanziger action on a background $B_{\mu}$, with the corresponding non-local horizon term $H(A, B)$ in (91), (93) and the generating functional $Z_{H}(B, J)$ in (90). However, since the background introduction (2), (19) has been applied to a non-local composite field it is natural to examine the consistency of the above interpretation with the symmetries exhibited by $Z_{H}(B, J)$. To this end, it is useful to recast $Z_{H}(B, J)$ in a local form by extending the configuration space according to [97], in such a way that one introduces a set of commuting $\left(\bar{\varphi}_{\mu}^{p q}, \varphi_{\mu}^{p q}\right)$ and anticommuting $\left(\bar{\omega}_{\mu}^{p q}, \omega_{\mu}^{p q}\right)$ auxiliary fields, where $\bar{\varphi}_{\mu}^{p q}$ and $\varphi_{\mu}^{p q}$ are mutually complex-conjugate,

$$
\epsilon\left(\bar{\varphi}_{\mu}^{p q}, \varphi_{\mu}^{p q}, \bar{\omega}_{\mu}^{p q}, \omega_{\mu}^{p q}\right)=(0,0,1,1), \operatorname{gh}\left(\bar{\varphi}_{\mu}^{p q}, \varphi_{\mu}^{p q}, \bar{\omega}_{\mu}^{p q}, \omega_{\mu}^{p q}\right)=(0,0,-1,1) .
$$

This makes it possible to construct the parametrization

$$
\exp \left\{\hbar^{-1}[H(A, B)-H(0, B)]\right\}=\int d \bar{\varphi} d \varphi d \bar{\omega} d \omega \exp \left[-\hbar^{-1} S_{\gamma}(A, B ; \bar{\varphi} \varphi, \bar{\omega}, \omega)\right],
$$

where

$$
S_{\gamma}=\int d^{D} x\left[-\bar{\varphi}_{\mu}^{r p} K_{B}^{p q} \varphi^{r q \mid \mu}+\bar{\omega}_{\mu}^{r p} K_{B}^{p q} \omega^{r q \mid \mu}+i \gamma^{1 / 2} g f^{p r q} A^{r \mid \mu}\left(\bar{\varphi}_{\mu}^{p q}+\varphi_{\mu}^{p q}\right)\right],
$$

as we denote

$$
K_{B}^{p q} \varphi_{\mu}^{r q}(x)=\int d^{D} y K_{B}^{p q}(x, y) \varphi_{\mu}^{r q}(y), \quad K_{B}^{p q} \omega_{\mu}^{r q}(x)=\int d^{D} y K_{B}^{p q}(x, y) \omega_{\mu}^{r q}(y) .
$$

In the configuration space $\Phi=(\phi, \bar{\varphi}, \varphi, \bar{\omega}, \omega)$, the functional $Z_{H}(B, J)$ of (90) takes the form

$$
Z_{H}(B, J)=\int d \Phi \exp \left\{-\hbar^{-1}\left[S_{\mathrm{GZ}}(\Phi, B)+J_{A} \phi^{A}\right]\right\},
$$

where the local action $S_{\mathrm{GZ}}(\Phi, B)$ of the Gribov-Zwanziger theory on a background reads (note the antisymmetry of $f^{p q r}$ )

$$
\begin{aligned}
S_{\mathrm{GZ}}(\Phi, B) & =S_{\mathrm{FP}}(\phi, B)-H(0, B)+S_{K}(\Phi, B)-i \gamma^{1 / 2} g \int d^{D} x \operatorname{Tr} A^{\mu}\left(\bar{\varphi}_{\mu}-\varphi_{\mu}^{\mathrm{T}}\right), \\
S_{K}(\Phi, B) & \equiv \int d^{D} x d^{D} y \operatorname{Tr}\left[-\bar{\varphi}^{\mu}(x) K_{B}(x, y) \varphi_{\mu}^{\mathrm{T}}(y)+\bar{\omega}^{\mu}(x) K_{B}(x, y) \omega_{\mu}^{\mathrm{T}}(y)\right] .
\end{aligned}
$$

Using the explicit form of $K_{B}(x, y)$ in (93), the antisymmetry of $f^{p q r}$, and a repeated integration by parts, one can remove the delta-function $\delta(x-y)$ absorbed in $K_{B}(x, y)$ and present $S_{K}(\Phi, B)$ in (98) as follows:

$$
S_{K}(\Phi, B)=\int d^{D} x \operatorname{Tr}\left[-\bar{\varphi}^{\mu} D_{v}(A+B) D^{v}(B) \varphi_{\mu}^{\mathrm{T}}+\bar{\omega}^{\mu} D_{v}(A+B) D^{v}(B) \omega_{\mu}^{\mathrm{T}}\right] .
$$


The action $S_{\mathrm{GZ}}(\Phi, B)$ is invariant under the global $S U(N)$ transformations

$$
\left(A_{\mu}, B_{\mu}, b, \bar{c}, c, \bar{\varphi}_{\mu}, \varphi_{\mu}^{\mathrm{T}}, \bar{\omega}_{\mu}, \omega_{\mu}^{\mathrm{T}}\right) \stackrel{U}{\rightarrow} U\left(A_{\mu}, B_{\mu}, b, \bar{c}, c, \bar{\varphi}_{\mu}, \varphi_{\mu}^{\mathrm{T}}, \bar{\omega}_{\mu}, \omega_{\mu}^{\mathrm{T}}\right) U^{-1} .
$$

The infinitesimal form of (100)

$$
\delta_{\varsigma} F^{p q}=g f^{p r s} F^{r q} \varsigma^{s}+g f^{q r s} F^{p r} \varsigma^{s}, \quad F^{p q}=\left(A_{\mu}, B_{\mu}, b, \bar{c}, c, \bar{\varphi}_{\mu}, \varphi_{\mu}^{\mathrm{T}}, \bar{\omega}_{\mu}, \omega_{\mu}^{\mathrm{T}}\right)^{p q}
$$

produces a unit Jacobian (due to the antisymmetry of $f^{p q r}$ ) in the integration measure of (97) and leaves the functional $Z_{H}(B, J)$ invariant under infinitesimal global $S U(N)$ transformations for the background field $B_{\mu}$ and the sources $J_{A}$, having the adjoint representation form

$$
\left.\delta_{\zeta} G^{p q}=g f^{p r s} G^{r q} \varsigma^{s}+g f^{q r s} G^{p r} \varsigma^{s}, \quad G^{p q}=\left(B_{\mu}, J_{\mu(A)}, J_{(b)}, J_{(\bar{c})}, J_{(c)}\right)\right)^{p q} .
$$

This behavior of $Z_{H}(B, J)$ obviously entails an invariance of the restricted generating functional $Z_{H}(J)$ under the global $S U(N)$ transformations of the sources; however, the global $S U(N)$ invariance of $Z_{H}(J)$ fails to translate itself into a local symmetry of the background functional $Z_{H}(J, B)$. Indeed, let us apply an infinitesimal form of the local change of variables $(U \rightarrow V)$

$$
\Phi \stackrel{V}{\rightarrow} \Phi^{\prime}, \quad B_{\mu} \stackrel{V}{\rightarrow} B_{\mu}^{\prime}=V B_{\mu} V^{-1}+g^{-1} V\left(\partial_{\mu} V^{-1}\right),
$$

with a unitary matrix $V=V(\xi), \xi=\xi(x)$, to the integrand in (97), which produces a unit Jacobian and a variation $\delta_{\xi} S_{\mathrm{GZ}}=\delta_{\xi} S_{K}$ in (98), so that the presence of extra derivatives $\partial_{\mu} V^{-1}$ and $\partial^{2} V^{-1}$ in the transformed expression

$$
\begin{aligned}
S_{K}\left(\Phi^{\prime}, B^{\prime}\right) & =\int d^{D} x \operatorname{Tr}\left[-V \bar{\varphi}^{\mu} D_{v}(A+B) D^{v}(B) \varphi_{\mu}^{\mathrm{T}} V^{-1}+V \bar{\omega}^{\mu} D_{v}(A+B) D^{v}(B) \omega_{\mu}^{\mathrm{T}} V^{-1}\right] \\
& \neq \int d^{D} x \operatorname{Tr}\left[-V^{-1} V \bar{\varphi}^{\mu} D_{v}(A+B) D^{v}(B) \varphi_{\mu}^{\mathrm{T}}+V^{-1} V \bar{\omega}^{\mu} D_{v}(A+B) D^{v}(B) \omega_{\mu}^{\mathrm{T}}\right],
\end{aligned}
$$

implies $S_{K}\left(\Phi^{\prime}, B^{\prime}\right) \neq S_{K}(\Phi, B)$, also involving a local non-invariance of the background horizon term, $H\left(A^{\prime}, B^{\prime}\right) \neq H(A, B)$. Finally, in view of $\delta_{\xi}\left(J_{A} \phi^{A}\right)=0$, the functional $Z_{H}(B, J)$ is not invariant under the background gauge transformations of $B_{\mu}$ with the local $S U(N)$ transformations of $J_{A}$, since the latter do not compensate the variation $\delta_{\xi} S_{\mathrm{GZ}}(\Phi, B) \neq 0$.

\subsection{Modified Background Introduction and Effective Action}

The local non-invariance of $Z_{H}(B, J)$ can be explained by the fact that the background field $B_{\mu}$ has been introduced directly into the non-local horizon term $H(A)$ by means of (93), while localizing the resultant background term $H(A, B)$ by the auxiliary fields $\left(\bar{\varphi}_{\mu}, \varphi_{\mu}\right)$ and $\left(\bar{\omega}_{\mu}, \omega_{\mu}\right)$ does not provide them with a covariant derivative as in (19), which is evident from (99). In order to remedy this issue, we examine an alternative introduction of a background field, i.e., by using a local parametrization of the original horizon term $H(A)$ at a point before a background has been switched on. To this end, let us consider the expressions (95)-(98) when restricted to $B_{\mu}=0$ and recast the functional $Z_{H}(J)$ given by (81) in the form

$$
Z_{H}(J)=\int d \Phi \exp \left\{-\hbar^{-1}\left[S_{\mathrm{GZ}}(\Phi)+J_{A} \phi^{A}\right]\right\}, S_{\mathrm{GZ}}(\Phi)=S_{\mathrm{GZ}}(\Phi, 0), \Phi=(\phi, \bar{\varphi}, \varphi, \bar{\omega}, \omega) .
$$


For a consideration of the auxiliary fields $(\bar{\varphi}, \bar{\omega})$ and $\left(\varphi^{\mathrm{T}}, \omega^{\mathrm{T}}\right)$ on equal terms, note that the action $S_{\mathrm{GZ}}(\Phi)$ in the above integrand with the Landau gauge condition $\partial_{\mu} A^{\mu}=0$ encoded in $\exp \left[-\hbar S_{\mathrm{FP}}(\phi)\right]$ is equivalent to an action $\mathcal{S}_{\mathrm{GZ}}(\Phi)$ which arises from replacing $K(x, y)$ by $\mathcal{K}(x, y)$ defined as

$$
\mathcal{K}(x, y) \equiv \frac{1}{2}[K(x, y)+\tilde{K}(x, y)], \quad \tilde{K}(x, y)=K(x, y)+g\left[\partial_{\mu}, A^{\mu}\right] \delta(x-y) .
$$

Using integration by parts, permutations under the sign of $\mathrm{Tr}$, and the Landau gauge condition in the path integral, one brings the equivalent action $\mathcal{S}_{\mathrm{GZ}}(\Phi)$ to the form

$$
\begin{aligned}
\mathcal{S}_{\mathrm{GZ}}(\Phi) & =S_{\mathrm{FP}}(\phi)-H(0)+S_{\mathcal{K}}(\Phi)-i \gamma^{1 / 2} g \int d^{D} x \operatorname{Tr} A^{\mu}\left(\bar{\varphi}_{\mu}-\varphi_{\mu}^{\mathrm{T}}\right), \\
S_{\mathcal{K}}(\Phi) & \equiv \frac{1}{2} \int d^{D} x \operatorname{Tr}\left\{\left[D^{v}(A), \bar{\varphi}^{\mu}\right] \partial_{\nu} \varphi_{\mu}^{\mathrm{T}}+\left(\partial^{v} \bar{\varphi}^{\mu}\right)\left[D_{\nu}(A), \varphi_{\mu}^{\mathrm{T}}\right]\right\}-(\bar{\varphi}, \varphi \rightarrow \bar{\omega}, \omega),
\end{aligned}
$$

which invites a natural introduction of a background, $\mathcal{S}_{\mathrm{GZ}}(\Phi) \rightarrow \mathcal{S}_{\mathrm{GZ}}(\Phi, B)$, according to (19),

$$
\begin{aligned}
\mathcal{S}_{\mathrm{GZ}}(\Phi, B)= & S_{\mathrm{FP}}(\phi, B)-H(0)+S_{\mathcal{K}}(\Phi, B)-i \gamma^{1 / 2} g \int d^{D} x \operatorname{Tr} A^{\mu}\left(\bar{\varphi}_{\mu}-\varphi_{\mu}^{\mathrm{T}}\right), \\
S_{\mathcal{K}}(\Phi, B)= & \frac{1}{2} \int d^{D} x \operatorname{Tr}\left\{\left[D^{v}(A+B), \bar{\varphi}^{\mu}\right]\left[D_{\nu}(B), \varphi_{\mu}^{\mathrm{T}}\right]+\left[D^{\nu}(B), \bar{\varphi}^{\mu}\right]\left[D_{\nu}(A+B), \varphi_{\mu}^{\mathrm{T}}\right]\right\} \\
& -(\bar{\varphi}, \varphi \rightarrow \bar{\omega}, \omega) \equiv \int d^{D} x\left(-\bar{\varphi}_{\mu}^{p q} \mathcal{K}_{B}^{p q \mid r s} \varphi^{r s \mid \mu}+\bar{\omega}_{\mu}^{p q} \mathcal{K}_{B}^{p q \mid r s} \omega^{r s \mid \mu}\right) .
\end{aligned}
$$

Let us consider the expression

$\langle F, G\rangle \equiv-\frac{1}{2} \int d^{D} x \operatorname{Tr}\left\{\left[D_{\mu}(A+B), F\right]\left[D^{\mu}(B), G\right]+\left[D_{\mu}(B), F\right]\left[D^{\mu}(A+B), G\right]\right\}, \epsilon(F)=\epsilon(G)$, and present it in the form

$$
\langle F, G\rangle=\int d^{D} x F^{p q} \mathcal{K}_{B}^{p q \mid r s} G^{r s}=\int d^{D} x d^{D} y F^{p q}(x) \mathcal{K}_{B}^{p q \mid r s}(x ; y) G^{r s}(y) .
$$

Then, due to the (anti)symmetry of $\langle F, G\rangle$ under the replacement $F \leftrightarrow G$, we find

$$
\mathcal{K}_{B}^{p q \mid r s}(x ; y)=\mathcal{K}_{B}^{r s \mid p q}(y ; x) .
$$

Given the above, we interpret $\mathcal{S}_{\mathrm{GZ}}(\Phi, B)$ in (106) as an alternative local Gribov-Zwanziger action on the background $B_{\mu}$, with the corresponding modified background horizon term $\mathcal{H}(A, B)$ defined as

$$
\exp \left\{\hbar^{-1}[\mathcal{H}(A, B)-\mathcal{H}(0, B)]\right\}=\int d \bar{\varphi} d \varphi d \bar{\omega} d \omega \exp \left[-\hbar^{-1} \mathcal{S}_{\gamma}(\Phi, B)\right], \mathcal{H}(0, B) \equiv H(0),
$$

where

$$
\begin{aligned}
\mathcal{S}_{\gamma}(\Phi, B) & =S_{\mathcal{K}}(\Phi, B)-i \gamma^{1 / 2} g \int d^{D} x \operatorname{Tr} A^{\mu}\left(\bar{\varphi}_{\mu}-\varphi_{\mu}^{T}\right) \\
& =\int d^{D} x\left[-\bar{\varphi}_{\mu}^{p q} \mathcal{K}_{B}^{p q \mid r s} \varphi^{r s \mid \mu}+\bar{\omega}_{\mu}^{p q} \mathcal{K}_{B}^{p q \mid r s} \omega^{r s \mid \mu}+i \gamma^{1 / 2} g f^{p r q} A^{r \mid \mu}\left(\bar{\varphi}_{\mu}^{p q}+\varphi_{\mu}^{p q}\right)\right] .
\end{aligned}
$$

By construction, the action $\mathcal{S}_{\mathrm{GZ}}(\Phi, B)$ in (106) is invariant under the local transformations (103), which implies a unit Jacobian in the infinitesimal case and leads to an invariance of the background generating functional

$$
Z_{\mathcal{H}}(B, J)=\int d \Phi \exp \left\{-\hbar^{-1}\left[\mathcal{S}_{\mathrm{GZ}}(\Phi, B)+J_{A} \Phi^{A}\right]\right\}, \quad Z_{\mathcal{H}}(0, J)=Z_{H}(J),
$$


with respect to local transformations of the sources and the background field:

$$
\delta_{\xi} B_{\mu}^{p}=D_{\mu}^{p q}(B) \xi^{q}, \quad \delta_{\xi}\left(J_{\mu(A)}, J_{(b)}, J_{(\bar{c})}, J_{(c)}\right)^{p}=g f^{p r q}\left(J_{\mu(A)}, J_{(b)}, J_{(\bar{c})}, J_{(c)}\right)^{r} \xi^{q} .
$$

The transformations (103) also provide a local invariance of the modified background horizon term $\mathcal{H}(A, B)$ in (107),

$$
\delta_{\xi} \mathcal{H}(A, B)=0, \quad \delta_{\xi} B_{\mu}^{p}=D_{\mu}^{p q}(B) \xi^{q}, \quad \delta_{\xi} A_{\mu}^{p}=g f^{p r q} A_{\mu}^{r} \xi^{q} .
$$

Introducing the generating functionals of connected $W_{\mathcal{H}}(B, J)$ and vertex $\Gamma_{\mathcal{H}}(B, \phi)$ Green's functions on a background,

$$
Z_{\mathcal{H}}=\exp \left(-\hbar^{-1} W_{\mathcal{H}}\right), \Gamma_{\mathcal{H}}(B, \phi)=W_{\mathcal{H}}(B, J)-J_{A} \phi^{A}, \phi^{A}=\frac{\vec{\delta}}{\delta J_{A}} W_{\mathcal{H}}, J_{A}=-\Gamma_{\mathcal{H}} \frac{\overleftarrow{\delta}}{\delta \phi^{A}}
$$

one translates the invariance of $Z_{\mathcal{H}}(B, J)$ under (109) into an invariance of $\Gamma_{\mathcal{H}}(B, \phi)$ under the following local transformations, cf. (22), (23), (34), (36),

$$
\delta_{\xi} B_{\mu}^{p}=D_{\mu}^{p q}(B) \xi^{q}, \quad \delta_{\xi}\left(A_{\mu}, b, \bar{c}, c\right)^{p}=g f^{p r q}\left(A_{\mu}, b, \bar{c}, c\right)^{r} \xi^{q},
$$

which consist of the gauge transformations for the background field $B_{\mu}$ and of the local $S U(N)$ transformations for the quantum fields $\phi^{A}$, so that the background effective action $\Gamma_{\text {eff }}(B)$ for the Gribov-Zwanziger model defined as

$$
\Gamma_{\text {eff }}(B)=\left.\Gamma_{\mathcal{H}}(B, \phi)\right|_{\phi=0}
$$

is invariant, $\delta_{\xi} \Gamma_{\text {eff }}=0$, under the gauge transformations of the background field $B_{\mu}$.

\section{Conclusions}

In the present article, we have approached the issue of a joint introduction of composite and background fields into non-Abelian quantum gauge models on the basis of symmetries exhibited by the generating functional of Green's functions. Thus, we examine the Yang-Mills theory, the Volovich-Katanaev model of two-dimensional gravity with dynamical torsion, and the Gribov-Zwanziger theory.

Our systematic analysis of the problem focuses on quantum Yang-Mills theories and local composite fields. As our first approach, we consider a theory quantized according to Faddeev and Popov and including some composite fields (1), with the resultant generating functional $Z(J, L)$ having an inherent symmetry under global $S U(N)$ transformations of the sources $J_{A}, L_{m}$ for the quantum $\phi^{A}$ and composite $\sigma^{m}(\phi)$ fields. As our second approach, we examine a theory quantized in the background field method (3), with the background generating functional $Z(B, J)$ being inherently invariant under local $S U(N)$ transformations of the sources $J_{A}$ accompanied by gauge transformations of the background field $B_{\mu}$ with an associated covariant derivative $D_{\mu}(B)$. In the first approach, we introduce a background field $B_{\mu}$ equipped with $D_{\mu}(B)$, whereas in the second approach we introduce some composite fields $\sigma^{m}(\phi, B)$ with sources $L_{m}$. In doing so, we demand that the functional $Z(B, J, L)$ given by $Z(J, L) \stackrel{B}{\rightarrow} Z(B, J, L)$ and $Z(B, J) \stackrel{L}{\rightarrow} Z(B, J, L)$ reflect the symmetries inherent in $Z(J, L)$ and $Z(B, J)$. The resultant $Z(B, J, L)$ is thereby invariant under the local $S U(N)$ transformations of $J_{A}, L_{m}$ combined with the gauge transformations of $B_{\mu}$, whereas our first and second approaches are equivalent in the sense of (2) and (4), (5) with $\partial_{\mu} \rightarrow D_{\mu}$ (B) given by (19).

In quantum Yang-Mills theories with composite and background fields, we have introduced an extended (due to antifields $\phi_{A}^{*}$ ) generating functional of vertex Green's functions (24), (29), including a background effective action (33), and investigated its properties. For the generating functional of 
vertex Green's functions (effective action), gauge dependence has been obtained in terms of a nilpotent operator with composite and background fields (30), (31), and conditions of on-shell independence from gauge-fixing have been established. Thus, the generating functional of vertex functions is found to be gauge-independent on its extremals (32), whereas the background effective action is is independent of any specific choice of gauge-fixing at the extremals (32) restricted to the hypersurface of vanishing antifields $\phi_{A}^{*}$ and quantum fields $\phi^{A}$. The background effective action $\Gamma_{\text {eff }}(B, \Sigma)$, depending on the background field $B_{\mu}$ and a set of auxiliary fields $\Sigma^{m}$ associated with the composite fields $\sigma^{m}$, is found to be invariant under the gauge transformations of $B_{\mu}$ and the local $S U(N)$ transformations of $\Sigma^{m}$, see (36).

Introduction of composite fields in the approach $Z(B, J) \stackrel{L}{\rightarrow} Z(B, J, L)$ starting from a background generating functional $Z(B, J)$ has been extended beyond the Yang-Mills case by considering the Volovich-Katanaev model [50] of two-dimensional gravity with dynamical torsion (in terms of a zweibein $e_{\mu}^{i}$ and a Lorentz connection $\omega_{\mu}$ ) quantized using the background field method in [75] and exhibiting a gauge-invariant background effective action. Thus, the quantum theory [75] has been modified by introducing local composite fields $\sigma_{\mu_{1} \cdots \mu_{n}}^{i_{1} \cdots i_{m}}$ with sources $L_{i_{1} \cdots i_{m}}^{\mu_{1} \cdots \mu_{n}}$, as we demand for $\sigma_{\mu_{1} \cdots \mu_{n}}^{i_{1} \cdots i_{m}}$ to behave as tensors (70) under the Lorentz (63) and general coordinate (64) transformations of background $B=\left(e_{\mu}^{i}, \omega_{\mu}\right)$ and quantum $Q=\left(q_{\mu}^{i}, q_{\mu}\right)$ fields. These transformations coincide infinitesimally with the background transformations $\delta_{\mathrm{b}} B$ and $\delta_{\mathrm{b}} Q$ in (53), constructed by analogy with the Yang-Mills case (44). The corresponding background effective action $\Gamma_{\text {eff }}(B, \Sigma)$ defined as (69), (74), (75) has been found invariant under the gauge transformations (49), (51) of background fields $B$ combined with the local transformations (76) of additional fields $\Sigma_{\mu_{1} \cdots \mu_{n}}^{i_{1} \cdots i_{m}}$ related to $\sigma_{\mu_{1} \cdots \mu_{n}}^{i_{1} \cdots i_{m}}$. As in the Yang-Mills case, (76) is an infinitesimal form of tensor transformations, cf. (70), (71).

Introduction of background fields in the approach $Z(J, L) \stackrel{B}{\rightarrow} Z(B, J, L)$ starting from a generating functional $Z(J, L)$ has been extended beyond the case of local composite fields by considering the Gribov-Zwanziger model [23,24] implementing the Gribov horizon [22] for quantum Yang-Mills theories in Landau gauge by means of an additive horizon functional $H(A)$ which can be presented in terms of a non-local composite field $\sigma(A)$. Direct introduction of a background field $B_{\mu}$ into the composite field, $\sigma(A) \rightarrow \sigma(A, B)$, produces a formally consistent (94) background-modified generating functional for the Gribov-Zwanziger theory, see $Z_{H}(B, J)$ in (90) with the corresponding non-local horizon term $H(A, B)$ in (91), (93). However, it has been shown that $Z_{H}(B, J)$ does not inherit (in a localized form) the symmetry of the original Gribov-Zwanziger functional $Z_{H}(J)$ in (81) under global $S U(N)$ transformations of the sources, as $H(A, B)$ fails to be invariant under local $S U(N)$ transformations of quantum Yang-Mills field $A_{\mu}$ combined with gauge transformations of the background field $B_{\mu}$. Further, representing the original horizon term $H(A)$ in a local parametrization using auxiliary fields according to [97], we bring the related functional $Z_{H}(J)$ to an equivalent (under the Landau gauge) form with a modified horizon term $\mathcal{H}(A)$. This local parametrization admits a natural introduction of a covariant derivative $D_{\mu}(B)$ as acting on the auxiliary fields by extension $\partial_{\mu} \rightarrow D_{\mu}(B)$ according to (19), which implies a background horizon term $\mathcal{H}(A, B)$ invariant under the local $S U(N)$ transformations of $A_{\mu}$ along with the gauge transformations of $B_{\mu}$. The resulting modified background functional $Z_{\mathcal{H}}(B, J)$ in (108) is consistent, $\left.Z_{\mathcal{H}}(B, J)\right|_{B=0}=Z_{H}(J)$, and invariant under the local $S U(N)$ transformations of the sources combined with the gauge transformations of the background field. The corresponding background effective action $\Gamma_{\text {eff }}(B)$ for the Gribov-Zwanziger model defined as (106), (108), (110), (112) proves to be invariant under the gauge transformations $\delta_{\xi} B_{\mu}=\left[D_{\mu}(B), \xi\right]$ of the background field $B_{\mu}$. This effective action seems advantageous as a starting point in approaching a renormalization analysis of the Gribov-Zwanziger model as one accounts for the horizon in the background field method.

One should note, the calculations maybe independently done in the Computer Algebra Systems, like Mathematica Singular or Maple. We checked the correctness some of the equations with use of the Mathematica. 
One more application of the suggested approach may be developed for QCD-gauge theory of strong interactions with $S U(3)$ gauge group [33,88]-to describe hadron (meson and baryon. e.g., proton, neutron) particles as the composite fields, $\sigma^{m}\left(\bar{u}, u, \bar{d}, d, A_{\mu}^{\bar{\alpha}}\right)$, of up $u$ and down $d$ quarks (including leftan right-handed spinors) of spin $1 / 2$ and $A_{\mu}^{\bar{\alpha}}, \bar{\alpha}=1, \ldots, 8$ of spin 1 gluons fields. Here, the Grassmann parity $\epsilon\left(\sigma^{m}\right)$ should equal to 1 for baryon and to 0 for meson particles.

Another interesting application of the presented background field method with composite fields is a consideration of so-called Generalized Lagrange space (for metric fields) to exploit its properties of curvature, torsion and deflection in order to take into account the asymmetries and anisotropies arising from physical phenomena basically at the cosmological level.

Author Contributions: Conceptualization, P.Yu.M. and A.A.R.; methodology, P.Yu.M. and A.A.R.; software, P.Yu.M., A.A.R.; validation, P.Yu.M., A.A.R.; formal analysis, P.Yu.M., A.A.R.; investigation, P.Yu.M., A.A.R.; resources, P.Yu.M., A.A.R.; data curation, P.Yu.M., A.A.R.; writing-original draft preparation, P.Yu.M.; writing一review and editing, P.Yu.M., A.A.R.; visualization, P.Yu.M., A.A.R.; supervision, P.Yu.M., A.A.R.; project administration, A.A.R.; funding acquisition, P.Yu.M., A.A.R. All authors have read and agreed to the published version of the manuscript.

Funding: This research received no external funding.

Acknowledgments: The work of P.Yu.M. has been supported by the Tomsk State University Competitiveness Improvement Program. A.A.R. is grateful for the support from the Program of Fundamental Research under the Russian Academy of Sciences, 2013-2020.

Conflicts of Interest: The authors declare no conflict of interest.

\section{References}

1. Cornwall, J.M.; Jackiw, R.; Tomboulis, E. Effective Action for Composite Operatorps. Phys. Rev. D 1974, 10, 2428-2445. [CrossRef]

2. Haymaker, R.W. Variational Methods for Composite Operators. Rivista Nuovo Cim. 1991, 14, 1-89. [CrossRef]

3. DeWitt, B.S. Quantum Theory of Gravity. II. The Manifestly Covariant Theory. Phys. Rev. 1967, 162, 1195-1239. [CrossRef]

4. Aref'eva, I.Y.; Faddeev, L.D.; Slavnov, A.A. Generating Functional for the S Matrix in Gauge Theories. Theor. Math. Phys. 1975, 21, 1165-1172, (in Russian: Teor. Mat. Fiz. 1974, 21, 311-321). [CrossRef]

5. Abbott, L.F. The Background Field Method beyond One Loop. Nucl. Phys. B 1981, 185, 189-203. [CrossRef]

6. Nambu, Y.; Jona-Lasinio, G. Dynamical Model of Elementary Particles Based on an Analogy with Superconductivity. Phys. Rev. 1961, 122, 345-358. [CrossRef]

7. Gross, D.; Neveu, A. Dynamical Symmetry Breaking in Asymptotically Free Field Theories. Phys. Rev. D 1974, 10, 3235-3253. [CrossRef]

8. Hawking, S.W.; Moss, I.G. Fluctuations in the Inflationary Universe. Nucl. Phys. B 1983, 224, 180-192. [CrossRef]

9. Casalbuoni, R.; DeCurtis, S.; Dominici, D.; Gatto, R. Composite Operator Calculation of Chiral Symmetry Breaking in Color Gauge Theory. Phys. Lett. B 1984, 140, 357-362. [CrossRef]

10. Bardeen, W.; Hill, C.; Lindner, M. Minimal Dynamical Symmetry Breaking of the Standard Model. Phys. Rev. D 1990, 41, 1647-1660. [CrossRef]

11. Grigorian, R.P.; Tyutin, I.V. Renormalization Group Equation for Composite Fields. Sov. J. Nucl. Phys. 1977, 26, 593, (in Russian: Yad. Fiz. 1977, 26, 1121-1129).

12. Gusynin, V.P.; Miransky, V.A. Nonperturbative Scale Anomaly and Composite Operators in Gauge Field Theories. Phys. Lett. 1987, B198, 362-366. [CrossRef]

13. Seiberg, N. The Power of Duality-Exact Results in 4D SUSY Field Theory. Int. J. Mod. Phys. A 2001, 16, 4365-4376. [CrossRef]

14. Shifman, M.; Stepanyantz, K. Exact Adler Function in Supersymmetric QCD. Phys. Rev. Lett. 2015, 114, 051601. [CrossRef]

15. Shifman, M.; Stepanyantz, K.V. Derivation of the Exact Expression for the D Function in $N=1$ SQCD. Phys. Rev. D 2015, 91, 105008. [CrossRef]

16. Capri, M.A.L.; van Egmond, D.M.; Guimarães, M.S.; Holanda, O.; Sorella, S.P.; Terin R.C.; Toledo H.C. Renormalizability of $N=1$ Super Yang-Mills Theory in Landau Gauge with a Stueckelberg-like Field. Eur. Phys. J. C2018, 78, 797. [CrossRef] 
17. Wetterich, C. Average Action and the Renormalization Group Equations. Nucl. Phys. B 1991, 352, 529-584. [CrossRef]

18. Reuter, M.; Wetterich, C. Average Action for the Higgs Model with Abelian Gauge Symmetry. Nucl. Phys. B 1993, 391, 147-175. [CrossRef]

19. Reuter, M.; Wetterich, C. Effective Average Action for Gauge Theories and Exact Evolution Equations. Nucl. Phys. B 1994, 417, 181-214. [CrossRef]

20. Lavrov, P.; Shapiro, I. On the Functional Renormalization Group Approach for Yang-Mills Fields. JHEP 2013, 1306, 086. [CrossRef]

21. Barra, V.F.; Lavrov, P.M.; dos Reis, E.A.; de Paula Netto, T.; Shapiro, I.L. Functional Renormalization Group Approach and Gauge Dependence in Gravity Theories. Phys. Rev. D 2020, 101, 065001. [CrossRef]

22. Gribov, V.N. Quantization of Nonabelian Gauge Theories. Nucl. Phys. B 1978, 139, 1-19. [CrossRef]

23. Zwanziger, D. Action from the Gribov Horizon. Nucl. Phys. B 1989, 321, 591-604. [CrossRef]

24. Zwanziger, D. Local and Renormalizable Action from the Gribov Horizon. Nucl. Phys. 1989, B323, 513-544. [CrossRef]

25. Lavrov, P.; Lechtenfeld, O.; Reshetnyak, A. Is Soft Breaking of BRST Symmetry consistent? JHEP 2011, 1110, 043. [CrossRef]

26. Reshetnyak, A. On Gauge Independence for Gauge Models with Soft Breaking of BRST Symmetry. Int. J. Mod. Phys. A 2014, 29, 1450184. [CrossRef]

27. Lavrov, P.; Radchenko, O.; Reshetnyak, A. Soft Breaking of BRST Symmetry and Gauge Dependence. Mod. Phys. Lett. A2012, 27, 1250067. [CrossRef]

28. Lavrov, P.; Lechtenfeld, O. Gribov Horizon beyond the Landau Gauge. Phys. Lett. B 2013, 725. [CrossRef]

29. Capri, M.A.L.; Fiorentini, D.; Guimaraes, M.S.; Mintz, B.W.; Palhares, L.F.; Sorella, S.P. A Local and Renormalizable Framework for the Gauge-invariant Operator $A_{\min }^{2}$ in Euclidean Yang-Mills Theories in Linear Covariant Gauges. Phys. Rev. D 2016, 94, 065009. [CrossRef]

30. Capri, M.A.L.; Sorella, S.P.; Terin, R.C. Study of a Gauge Invariant Local Composite Fermionic Field. Ann. Phys. 2020, 414, 168077. [CrossRef]

31. Canfora, F.E.; Hidalgo, D.; Pais, P. The Gribov Problem in Presence of Background Field for SU(2) Yang-Mills theory. Phys. Lett. B 2016, 763, 94-101. [CrossRef]

32. Abbott, L.F. Introduction to the Background Field Method. Acta Phys. Polon. B 1982, 13, 33-50.

33. Weinberg, S.; The Quantum Theory of Fields. Vol. II Modern Applications; Cambridge University Press: Cambridge, UK, 1996.

34. 't Hooft, G. An Algorithm for the Poles at Dimension four in the Dimensional Regularization Procedure. Nucl. Phys. B 1973, 62, 444-460. [CrossRef]

35. Kluberg-Stern, H.; Zuber, J.B. Renormalization of non-Abelian Gauge Theories in a Background-field Gauge. I. Green's Functions. Phys. Rev. D 1975, 12, 482-488. [CrossRef]

36. Grisaru, M.T.; van Nieuwenhuizen, P. ; Wu, C.C. Background-field Method versus Normal Field Theory in Explicit Examples: One Loop Divergences in S Matrix and Green's Functions for Yang-Mills and Gravitational Fields. Phys. Rev. D 1975, 12, 3203-3213. [CrossRef]

37. Capper, D.M.; MacLean, A. The Background Field Method at Two Loops: A General Gauge Yang-Mills Calculation. Nucl. Phys. B 1982, 203, 413-422. [CrossRef]

38. Ichinose, S.; Omote, M. Renormalization Using the Background-field Method. Nucl. Phys. B 1982, 203, 221-267. [CrossRef]

39. Goroff, M.H.; Sagnotti, A. The Ultraviolet Behavior of Einstein Gravity. Nucl. Phys. B1986, 266. [CrossRef]

40. van de Ven, A.E.M. Two-loop Quantum Gravity. Nucl. Phys. B 1992, 378, 309-366. [CrossRef]

41. Grassi, P.A. Algebraic Renormalization of Yang-Mills Theory with Background Field Method. Nucl. Phys. B 1996, 462, 524-550. [CrossRef]

42. Becchi, C.; Collina, R. Further Comments on the Background Field Method and Gauge Invariant Effective Actions. Nucl. Phys. B1999, 562, 412-430. [CrossRef]

43. Ferrari, R.; Picariello, M.; Quadri, A. Algebraic Aspects of the Background Field Method. Ann. Phys. 2001, 294, 165-181. [CrossRef]

44. Binosi, D.; Quadri, A. The Background Field Method as a Canonical Transformation. Phys. Rev. D 2012, 85, 121702. [CrossRef] 
45. Barvinsky, A.O.; Blas, D.; Herrero-Valea, M.; Sibiryakov, S.M.; Steinwachs, C.F. Renormalization of Gauge Theories in the Background-field Approach. JHEP 2018, 1807, 035. [CrossRef]

46. Frenkel, J.; Taylor, J.C. Background Gauge Renormalization and BRST Identities. Ann. Phys. 2018, 389, 234-238. [CrossRef]

47. Batalin, I.A.; Lavrov, P.M.; Tyutin, I.V. Multiplicative Renormalization of Yang-Mills Theories in the Background-field Formalism. Eur. Phys. J. C 2018, 78, 570. [CrossRef]

48. Brandt, F.T.; Frenkel, J.; McKeon, D.G.C. Renormalization of Six-dimensional Yang-Mills Theory in a Background Gauge Field. Phys. Rev. D 2019, 99, 025003. [CrossRef]

49. Faddeev, L.D.; Popov, V.N. Feynman Diagrams for the Yang-Mills Field. Phys. Lett. B 1967, 25, $29-30$. [CrossRef]

50. Katanaev, M.O.; Volovich, I.V. Two-dimensional Gravity with Dynamical Torsion and Strings. Ann. Phys. 1990, 197, 1-32. [CrossRef]

51. Jackiw, R. Lower Dimensional Gravity. Nucl. Phys. B1985, 252, 343-356. [CrossRef]

52. Teitelboim, C. Gravitation and Hamiltonian Structure in Two Space-Time Dimensions. Phys. Lett. B 1983, 126, 41-45. [CrossRef]

53. Banks, T.; Susskind, L. Canonical Quantization of 1+1 Dimensional Gravity. Int. J. Theor. Phys. 1984, 23, 475-496. [CrossRef]

54. Lichtzier, I.M.; Odintsov, S.D. Two-dimensional Quantum Gravity with Generalized Action and Compact Boson. Mod. Phys. Lett. A 1991, 6, 1953-1960. [CrossRef]

55. Sanchez, N. Semiclassical Quantum Gravity and Liouville Theory: A Complete Solution to the Back Reaction Problem in Two-dimensions. Nucl. Phys. B 1986, 266, 487-508. [CrossRef]

56. Brown, J.D.; Henneaux, M.; Teitelboim, C. Black Holes in Two Space-time Dimensions. Phys. Rev. D 1986, 33, 319-323. [CrossRef]

57. Balbinot, R.; Floreanini, R. Semiclassical Two-dimensional Gravity and Liouville Equation. Phys. Lett. 1985, B151, 401-404. [CrossRef]

58. Floreanini, R. On the Quantization of the Liouville Theory. Annals Phys. 1986, 167, 317-327. [CrossRef]

59. Fukuyama, T.; Kamimura, K. Gauge Theory of Two-dimensional Gravity. Phys. Lett. B 1985, 160, 259-262. [CrossRef]

60. Martellini, M. Some Remarks on the Liouville Approach to Two-dimensional Quantum Gravity. Ann. Phys. 1986, 167, 437-453. [CrossRef]

61. Marnelius, R. Canonical Quantization of Polyakov's String in Arbitrary Dimensions. Nucl. Phys. B 1983, 211, 14-28. [CrossRef]

62. Brink, L.; Di Vecchia, P.; Howe, P. A Locally Supersymmetric and Reparametrization Invariant Action for the Spinning String. Phys. Lett. 1976, B65, 471-474. [CrossRef]

63. Deser, S.; Zumino, B. A Complete Action for the Spinning String. Phys. Lett. B 1976,65, 369-373. [CrossRef]

64. Green, M.B.; Schwarz, J.D.; Witten, E. Superstring Theory; Cambridge University Press: London, UK, 1987.

65. Van Nieuwenhuizen, P. The Actions of the $N=1$ and $N=2$ Spinning Strings as Conformal Supergravities. Int. J. Mod. Phys. A1986, 1, 155-191. [CrossRef]

66. Katanaev, M.O. Conformal Invariance, Extremals, and Geodesics in Two-dimensional Gravity with Torsion. J. Math. Phys. 1991, 32, 2483-2496. [CrossRef]

67. Katanaev, M.O. All Universal Coverings of Two-dimensional Gravity with Torsion. 1993, 34, 700-736. [6C]

68. Kummer, W.; Schwarz, D.J. General Analytic Solution of $R^{2}$ Gravity with Dynamical Torsion in Two Dimensions Phys. Rev. D 1992, 45, 3628-3635. [CrossRef]

69. Solodukhin, S.N. Cosmological Solutions in 2D Poincaré Gravity. Int. J. Mod. Phys. D 1994, 3, 269-272. [CrossRef]

70. Strobl, T. All Symmetries of Non-Einsteinian Gravity in $d=2$. Int. J. Mod. Phys. A 1993, 8, 1383-1397. [CrossRef]

71. Schaller, P.; Strobl, T. Canonical Quantization of Non-Einsteinian Gravity and the Problem of Time. Class. Quant. Grav. 1994, 11, 331-346. [CrossRef]

72. Haider, F.; Kummer, W. Quantum Functional Integration of Non-Einsteinian Gravity in $d=2$. Int. J. Mod. Phys. A 1994, 9, 207-220. [CrossRef]

73. Kummer, W.; Schwarz, D.J. Renormalization of $R^{2}$ Gravity with Dynamical Torsion in $d=2$. Nucl. Phys. $B$ 1992, 382, 171-186. [CrossRef]

74. Ikeda, N., Izawa, K. Quantum Gravity with Dynamical Torsion in Two Dimensions. Prog. Theor. Phys. 1993, 89, 223-230. [CrossRef] 
75. Lavrov, P.M.; Moshin, P.Yu. Quantization of Two-dimensional Gravity with Dynamical Torsion. Class. Quant. Grav. 1999, 16, 2247-2258. [CrossRef]

76. Katanaev, M.O., Volovich, I.V. String Model with Dynamical Geometry and Torsion. Phys. Lett. B 1986, 175, 413-416. [CrossRef]

77. Reshetnyak, A. On Composite Fields Approach to Gribov Copies Elimination in Yang-Mills Theories. Phys. Part. Nucl. Lett. 2014, 11, 964-967. [CrossRef]

78. Joglekar, S.D.; Mandal, B.P. Finite Field Dependent BRS Transformations. Phys. Rev. D 1995, 51, $1919-1927$. [CrossRef]

79. Upadhyay, S.; Mandal, B.P., Field Dependent Nilpotent Symmetry for Gauge Theories Eur. Phys. J. C 2012, 72, 2065. [CrossRef]

80. Upadhyay, S.; Mandal, B.P.; Reshetnyak, A.A., Comments on Interactions in the SUSY Models. Eur. Phys. J. C 2016, 6, 391. [CrossRef]

81. Lavrov, P.; Lechtenfeld, O. Field-dependent BRST Transformations in Yang-Mills Theory. Phys. Lett. B 2013, 725, 382-385. [CrossRef]

82. Moshin, P.Yu.; Reshetnyak, A.A. Field-dependent BRST-antiBRST transformations in Yang-Mills and Gribov-Zwanziger theories. Nucl. Phys. B 2014, 888, 92-128. [CrossRef]

83. Moshin, P.Yu.; Reshetnyak, A.A. Finite BRST-antiBRST Transformations in Lagrangian Formalism. Phys. Lett. B 2014, 739, 110-116. [CrossRef]

84. Moshin, P.Yu.; Reshetnyak, A.A. Field-dependent BRST-antiBRST Lagrangian Transformations. Int. J. Mod. Phys. A 2015, 30, 1550021. [CrossRef]

85. Sobreiro, R.F.; Sorella, S.P. A Study of the Gribov Copies in Linear Covariant Gauges in Euclidean Yang-Mills Theories. JHEP 2005, 0506, 054. [CrossRef]

86. Capri, M.A.L.; Dudal, D.; Fiorentini, D.; Guimaraes, M.S.; Justo I.F.; Pereira A.D.; Mintz, B. W.; Palhares, L.F.; Sobreiro, R.F.; Sorella S.P. An Exact Nilpotent Nonperturbative BRST Symmetry for the Gribov-Zwanziger Action in the Linear Covariant Gauge. Phys. Rev. D 2015, 92, 045039. [CrossRef]

87. Capri, M.A.L.; Dudal, D.; Fiorentini, D.; Guimaraes, M.S.; Justo I.F.; Pereira A.D.; Mintz, B. W.; Palhares, L.F., Sobreiro, R. F.; Sorella S.P. A Local and BRST-invariant Yang-Mills Theory within the Gribov Horizon. Phys. Rev. D 2016, 94, 025035. [CrossRef]

88. Moshin, P.Y.; Reshetnyak, A.A. Finite Field-Dependent BRST-antiBRST Transformations: Jacobians and Application to the Standard Model. Int. J. Mod. Phys. A 2016, 31, 1650111. [CrossRef]

89. Moshin, P.Y.; Reshetnyak, A.A. On the Finite BRST Transformations: the Jacobians and the Standard Model with the Gauge-invariant Gribov Horizon. Russ. Phys. J. 2016, 59, 1921-1929. [CrossRef]

90. DeWitt, B.S. Dynamical Theory of Groups and Fields; Gordon and Breach: New York, NY, USA, 1965.

91. Becchi, C.; Rouet, A.; Stora, R. The Abelian Higgs-Kibble, Unitarity of the S-operator. Phys. Lett. B 1974, 52, 344-346. [CrossRef]

92. Becchi, C.; Rouet, A.; Stora, R. Renormalization of Gauge Theories. Ann. Phys. (N.Y.) 1976, 98, 287-321. [CrossRef]

93. Tyutin, I.V. Gauge Invariance in Field Theory and Statistical Physics in Operator Formalism; Lebedev Physical Institute: Moscow, Russia, 1975.

94. Lavrov, P.M.; Odintsov, S.D. The Gauge Dependence of the Effective Action of Composite Fields in General Gauge Theories. Int. J. Mod. Phys. A 1989, 4, 5205-5212. [CrossRef]

95. Lavrov, P.M.; Odintsov, S.D.; Reshetnyak, A.A. Effective Action of Composite Fields for General Gauge Theories in BLT-Covariant Formalism J. Math. Phys. 1997, 38, 3466-3477. [CrossRef]

96. Lavrov, P.M.; Odintsov, S.D.; Reshetnyak, A.A. Gauge Dependence in General Gauge Theories with Composite Fields in the Sp(2) Covariant Quantization Method Phys. At. Nuclei. 1997, 60, 1020. [CrossRef]

97. Kondo, K.-I. The Nilpotent "BRST" Symmetry for the Gribov-Zwanziger Theory; Report Number: CHIBA-EP-176; Chiba University: Chiba, Japan, 2009.

Publisher's Note: MDPI stays neutral with regard to jurisdictional claims in published maps and institutional affiliations. 